Algebraic $\&$ Geometric Topology

Volume 3 (2003) 287-334

Published: 13 March 2003

ATG

\title{
Equivalences of monoidal model categories
}

\author{
STEFAn SCHWEDE \\ BROOKE SHIPLEY
}

\begin{abstract}
We construct Quillen equivalences between the model categories of monoids (rings), modules and algebras over two Quillen equivalent model categories under certain conditions. This is a continuation of our earlier work where we established model categories of monoids, modules and algebras [SS00]. As an application we extend the Dold-Kan equivalence to show that the model categories of simplicial rings, modules and algebras are Quillen equivalent to the associated model categories of connected differential graded rings, modules and algebras. We also show that our classification results from [SS03] concerning stable model categories translate to any one of the known symmetric monoidal model categories of spectra.
\end{abstract}

AMS Classification 55U35; 18D10, 55P43, 55P62

Keywords Model category, monoidal category, Dold-Kan equivalence, spectra

\section{Introduction}

This paper is a sequel to [SS00] where we studied sufficient conditions for extending Quillen model category structures to the associated categories of monoids (rings), modules and algebras over a monoidal model category. Here we consider functors between such categories. We give sufficient conditions for extending Quillen equivalences of two monoidal model categories to Quillen equivalences on the associated categories of monoids, modules and algebras. This is relatively easy when the initial Quillen equivalence is via an adjoint pair of functors which induce adjoint functors on the categories of monoids; see for example [MMSS, $\S 13,16]$ and [Sch01, 5.1]. We refer to this situation as a strong monoidal Quillen equivalence, see Definition 3.6.

However, in the important motivating example of chain complexes and simplicial abelian groups, only something weaker holds: the right adjoint has a monoidal structure, but the left adjoint only has a lax comonoidal (also referred 
to as op-lax monoidal) structure which is a weak equivalence on cofibrant objects. We refer to this situation as a weak monoidal Quillen equivalence. Our general result about monoidal equivalences, Theorem 3.12, works under this weaker assumption. Also, in Proposition 3.16 we give a sufficient criterion for showing that an adjoint pair is a weak monoidal Quillen equivalence.

Our motivating example is the Dold-Kan equivalence of chain complexes and simplicial abelian groups. The normalization functor $N: s \mathcal{A} b \longrightarrow c h^{+}$is monoidal with respect to the graded tensor product of chains, the levelwise tensor product of simplicial abelian groups and the transformation known as the shuffle map; the inverse equivalence $\Gamma: c h^{+} \longrightarrow s \mathcal{A} b$ also has a monoidal structure (coming from the Alexander-Whitney map).

The natural isomorphism $N \Gamma \cong \mathrm{Id}_{c h^{+}}$is monoidal with respect to the shuffle and Alexander-Whitney maps. This implies that the algebra valued functor $\Gamma$ embeds connective differential graded rings as a full, reflexive subcategory of the category of simplicial rings, see Proposition 2.13. However, the other natural isomorphism $\Gamma N \cong \operatorname{Id}_{s \mathcal{A} b}$ cannot be chosen in a monoidal fashion. Hence these functors do not induce inverse, or even adjoint functors on the categories of algebras. One of the main points of this paper is to show that nevertheless, the homotopy categories of simplicial rings and connective differential graded rings are equivalent, via a Quillen equivalence on the level of model categories. This Quillen equivalence should be well known but does not seem to be in the literature. A similar equivalence, between reduced rational simplicial Lie algebras and reduced rational differential graded Lie algebras, was part of Quillen's work on rational homotopy theory [Qui69, I.4] which originally motivated the definition of model categories.

In the following theorem we use the word connective for non-negatively graded or more precisely $\mathbb{N}$-graded objects such as chain complexes or algebras.

Theorem 1.1 (1) For a connective differential graded ring $R$, there is a Quillen equivalence between the categories of connective $R$-modules and simplicial modules over the simplicial ring $\Gamma R$,

$$
\operatorname{Mod}-R \simeq_{Q} \operatorname{Mod}-\Gamma R .
$$

(2) For a simplicial ring $A$ there is a Quillen equivalence between the categories of connective differential graded NA-modules and simplicial modules over $A$,

$$
\operatorname{Mod}-N A \simeq_{Q} \operatorname{Mod}-A
$$


(3) For a commutative ring $k$, there is a Quillen equivalence between the categories of connective differential graded $k$-algebras and simplicial $k$ algebras,

$$
\mathcal{D} G A_{k} \simeq_{Q} \quad k-\mathcal{A l g} .
$$

(4) For $A$ a simplicial commutative ring, there is a Quillen equivalence between the categories of connective differential graded $N A$-algebras and simplicial $A$-algebras,

$$
N A-\mathcal{A l g} \simeq_{Q} \quad A-\mathcal{A l g}
$$

The special case for $k=\mathbb{Z}$ in part (3) of the previous theorem in particular says that the model categories of connective differential graded rings and simplicial rings are Quillen equivalent. Part (3) is a special case of part (4) for $A$ a constant commutative simplicial ring. The proof of Theorem 1.1 is an application of the more general Theorem 3.12. Parts (2) and (3) of Theorem 1.1 are established in Section 4.2, part (1) is shown in Section 4.3, and part (4) is completed in 4.4 .

In part (1) of Theorem 1.1, the right adjoint of the Quillen equivalence is induced by the functor $\Gamma$ from connective chain complexes to simplicial abelian groups which is inverse to normalized chain complex functor $N$. However, the left adjoint is in general not given by the normalized chain complex on underlying simplicial abelian groups. In parts (2) to (4), the right adjoint of the Quillen equivalence is always induced by the normalized chain complex functor. However, the left adjoint is in general not given by the functor $\Gamma$ on underlying chain complexes. We discuss the various left adjoints in Section 3.3.

Notice that we do not compare the categories of commutative simplicial rings and commutative differential graded rings. The normalization functor is symmetric monoidal with respect to the shuffle map. Hence it takes commutative simplicial rings to commutative (in the graded sense) differential graded rings. But the Alexander-Whitney map is not symmetric, and so $\Gamma$ does not induce a functor backwards. In characteristic zero, i.e., for algebras $k$ over the rational numbers, there is a model structure on commutative differential graded rings with underlying fibrations and trivial fibrations [BG76, St]. Moreover, the normalized chain complex functor is then the right adjoint of a Quillen equivalence between commutative simplicial $k$-algebras and connective differential graded $k$-algebras; indeed, as Quillen indicates on p. 223 of [Qui69], a similar method as for rational Lie algebras works for rational commutative algebras. 
Without a characteristic zero assumption, not every commutative differential graded ring is quasi-isomorphic to the normalization of a commutative simplicial ring: if $A$ is a commutative simplicial ring, then every element $x$ of odd degree in the homology algebra $H_{*}(N A)$ satisfies $x^{2}=0$; but in a general commutative differential graded algebra we can only expect the relation $2 \cdot x^{2}=0$. More generally, the homology algebra $H_{*}(N A)$, for $A$ a commutative simplicial ring, has divided power [Ca54] and other operations [Dw80] which need not be supported by a general commutative differential graded algebra. Moreover, in general the forgetful functor from differential graded algebras to chain complexes does not create a model structure and there is no homotopically meaningful way to go from differential graded to simplicial algebras in a way that preserves commutativity. While the normalization functor on commutative algebras still has a left adjoint, it is not clear if that adjoint preserves enough weak equivalences and whether it admits a derived functor.

In arbitrary characteristic, one should consider the categories of $E_{\infty}$-algebras instead of the commutative algebras. Mandell [Man, 1.2] establishes a Quillen equivalence, in any characteristic, between $E_{\infty}$-simplicial algebras and connective $E_{\infty}$-differential graded algebras. The symmetry properties of the Dold-Kan equivalence were also studied by Richter [Ri03]; she has shown that for every differential graded algebra $R$ which is commutative (in the graded sense), the simplicial ring $\Gamma A$ admits a natural $E_{\infty}$-multiplication.

One of the reasons we became interested in generalizing the Dold-Kan equivalence is because it is the basis for one out of the four steps of a zig-zag of weak monoidal Quillen equivalences between $H \mathbb{Z}$-modules, and $\mathbb{Z}$-graded chain complexes; see $[S]$. Theorem 3.12 then applies to each of these four steps to produce Quillen equivalences between $H \mathbb{Z}$-algebras and $\mathbb{Z}$-graded differential graded algebras and between the associated module categories [S, 1.1]. These equivalences then provide an algebraic model for any rational stable model category with a set of small generators. These rational algebraic models, [S, 1.2], are really unfinished business from [SS03] and even appeared in various preprint versions. These models are used as stepping stones in [Sh02] and [GS] to form explicit, small algebraic models for the categories of rational $T^{n}$-equivariant spectra for $T^{n}$ the $n$-dimensional torus.

Another motivation for this general approach to monoidal Quillen equivalences is the extension of our work in [SS03] where we characterize stable model categories with a set of generators as those model categories which are Quillen equivalent to modules over a symmetric ring spectrum with many objects ( $S p^{\Sigma_{\text {- }}}$ category). Here we show this characterization can be translated to any of 
the other symmetric monoidal categories of spectra. Quillen equivalences of monoids, modules and algebras for these categories of spectra were considered in [MMSS] and [Sch01], but ring spectra with many objects (or enriched categories) were not considered. Using the Quillen equivalences between modules over ring spectra with many objects (or enriched categories) over the other known highly structured categories of spectra established in Section 7, the characterization of [SS03, Thm 3.3.3] can be translated to any other setting:

Corollary 1.2 Let $\mathcal{C}$ be any of the monoidal model categories of symmetric spectra [HSS, MMSS] (over simplicial sets or topological spaces), orthogonal spectra [MMSS], $\mathcal{W}$-spaces [MMSS], simplicial functors [Lyd98] or $S$ modules $[E K M M]$. Then any cofibrantly generated, proper, simplicial, stable model category with a set of small generators is Quillen equivalent to modules over a $\mathcal{C}$-category with one object for each generator.

Organization In Section 2 we motivate our general results by considering the special case of chain complexes and simplicial abelian groups. We then turn to the general case and state sufficient conditions for extending Quillen equivalences to monoids, modules and algebras in Theorem 3.12. Section 3.4 gives a criterion for a Quillen functor pair to be weakly monoidal: a sufficient condition is that one of the unit objects detects weak equivalences. In Section 4 we return to chain complexes and simplicial abelian groups and deduce Theorem 1.1 from the general result. Section 5 contains the proof of the main theorem and the proof of the criterion for a Quillen functor pair to be weakly monoidal. In Section 6 we consider rings with many objects (enriched categories) and their modules. In Theorem 6.5 we extend the Quillen equivalences to modules over these enriched categories. In Section 7 we show that these general statements apply to the various symmetric monoidal categories of spectra and deduce Corollary 1.2. Throughout this paper, modules over a ring, algebra, category, etc, are always right modules.

Acknowledgments We would like to thank Mike Mandell for several helpful conversations. The second author was partially supported by an NSF Grant.

\section{Chain complexes and simplicial abelian groups}

As motivation for our general result, in this section we begin the comparison of the categories of differential graded rings and simplicial rings. We recall the normalized chain complex functor $N$, its inverse $\Gamma$ and the shuffle and 
Alexander-Whitney maps. We then consider the monoidal properties of the adjunction unit $\eta: \mathrm{Id} \longrightarrow \Gamma N$ and counit $\epsilon: N \Gamma \longrightarrow \mathrm{Id}$. As mentioned in the introduction, $\epsilon$ is monoidally better behaved than $\eta$. This motivates developing our general result in Section 3 which does not require monoidal adjunctions. In Section 4 we then revisit this specific example and prove Theorem 1.1.

\subsection{Normalized chain complexes}

The (ordinary) chain complex $C A$ of a simplicial abelian group $A$ is defined by $(C A)_{n}=A_{n}$ with differential the alternating sum of the face maps,

$$
d=\sum_{i=0}^{n}(-1)^{i} d_{i}:(C A)_{n} \longrightarrow(C A)_{n-1} .
$$

The chain complex $C A$ has a natural subcomplex $D A$, the complex of degenerate simplices; by definition, $(D A)_{n}$ is the subgroup of $A_{n}$ generated by all degenerate simplices. The normalized chain complex $N A$ is the quotient complex of $C A$ by the degenerate simplices,

$$
N A=C A / D A \text {. }
$$

The degenerate complex $D A$ is acyclic, so the projection $C A \longrightarrow N A$ is a quasi-isomorphism.

The complex of degenerate simplices has a natural complement, sometimes called the Moore complex. The $n$-th chain group of this subcomplex is the intersection of the kernels of all face maps, except the 0th one, and the differential in the subcomplex is thus given by the remaining face map $d_{0}$. The chain complex $C A$ is the internal direct sum of the degeneracy complex $D A$ and the Moore complex. In particular, the Moore subcomplex is naturally isomorphic to the normalized chain complex $N A$; in this paper, we do not use the Moore complex.

The normalization functor

$$
N: s \mathcal{A} b \longrightarrow c h^{+}
$$

from simplicial abelian groups to non-negatively graded chain complexes is an equivalence of categories [Do58, Thm. 1.9]. The value of the inverse $\Gamma: c h^{+} \longrightarrow$ $s \mathcal{A} b$ on a complex $C$ can be defined by

$$
(\Gamma C)_{n}=\operatorname{ch}^{+}\left(N \Delta^{n}, C\right),
$$

where $N \Delta^{n}$ is short for the normalized chain complex of the simplicial abelian group freely generated by the standard $n$-simplex. The simplicial structure 
maps in $\Gamma C$ are induced from the cosimplicial structure of $\Delta^{n}$ as $n$ varies through the simplicial category $\Delta$. A natural isomorphism

$$
\eta_{A}: A \longrightarrow \Gamma N A
$$

is defined in simplicial dimension $n$ by

$$
A_{n} \ni a \longmapsto\left(N \bar{a}: N \Delta^{n} \longrightarrow N A\right) \in(\Gamma N A)_{n}
$$

where $\bar{a}: \Delta^{n} \longrightarrow A$ is the unique morphism of simplicial sets which sends the generating $n$-simplex of $\Delta^{n}$ to $a \in A_{n}$. The other natural isomorphism $\epsilon_{C}: N \Gamma C \longrightarrow C$ is uniquely determined by the property

$$
\Gamma\left(\epsilon_{C}\right)=\eta_{\Gamma C}^{-1}: \Gamma N \Gamma C \longrightarrow \Gamma C .
$$

\section{$2.2 \quad$ Tensor products}

The tensor product of two connective chain complexes $C$ and $D$ is defined by

$$
(C \otimes D)_{n}=\bigoplus_{p+q=n} C_{p} \otimes D_{q}
$$

with differential given on homogeneous elements by

$$
d(x \otimes y)=d x \otimes y+(-1)^{|x|} x \otimes d y .
$$

The tensor product of simplicial abelian groups is defined dimensionwise.

Both tensor products are symmetric monoidal. The respective unit object is the free abelian group of rank one, viewed either as a complex concentrated in dimension zero or a constant simplicial abelian group. The associativity and unit isomorphisms are obvious enough that we do not specify them, similarly the commutativity isomorphism for simplicial abelian groups. The commutativity isomorphism for complexes involves a sign, i.e.,

$$
\tau_{C, D}: C \otimes D \longrightarrow D \otimes C
$$

is given on homogeneous elements by $\tau(x \otimes y)=(-1)^{|x||y|} y \otimes x$.

The unit objects are preserved under the normalization functor and its inverse. However, the two tensor products for chain complexes and simplicial abelian groups are different in an essential way, i.e., the equivalence of categories given by normalization does not take one tensor product to the other. Another way of saying this is that if we use the normalization functor and its inverse to transport the tensor product of simplicial abelian groups to the category of connective chain complexes, we obtain a second monoidal product (sometimes called the 
shuffle product of complexes) which is non-isomorphic, and significantly bigger than, the tensor product (2.3). Another difference is that the tensor product (2.3) makes perfect sense for $\mathbb{Z}$-graded chain complexes, whereas the shuffle product cannot be extended to a monoidal structure on $\mathbb{Z}$-graded chain complexes in any natural way. The difference between these two tensor products is responsible for the fact that the categories of simplicial rings and of connective differential graded rings are not equivalent.

\subsection{The shuffle and Alexander-Whitney maps}

Even though the tensor products of chain complexes and simplicial modules do not coincide under normalization, they can be related in various ways. The shuffle map

$$
\nabla: C A \otimes C B \longrightarrow C(A \otimes B),
$$

was introduced by Eilenberg and Mac Lane [EM53, (5.3)], see also [ML63, VIII 8.8] or [May67, 29.7]. For simplicial abelian groups $A$ and $B$ and simplices $a \in A_{p}$ and $b \in B_{q}$, the image $\nabla(a \otimes b) \in C_{p+q}(A \otimes B)=A_{p+q} \otimes B_{p+q}$ is given by

$$
\nabla(a \otimes b)=\sum_{(\mu, \nu)} \operatorname{sign}(\mu, \nu) \cdot s_{\nu} a \otimes s_{\mu} b
$$

here the sum is taken over all $(p, q)$-shuffles, i.e., permutations of the set $\{0, \ldots, p+q-1\}$ which leave the first $p$ elements and the last $q$ elements in their natural order. Such a $(p, q)$-shuffle is of the form $(\mu, \nu)=\left(\mu_{1}, \ldots, \mu_{p}, \nu_{1}, \ldots, \nu_{q}\right)$ with $\mu_{1}<\mu_{2}<\cdots<\mu_{p}$ and $\nu_{1}<\cdots<\nu_{q}$, and the associated degeneracy operators are given by

$$
s_{\mu} b=s_{\mu_{p}} \cdots s_{\mu_{1}} b \quad \text { respectively } \quad s_{\nu} a=s_{\nu_{q}} \cdots s_{\nu_{1}} a .
$$

The shuffle map is a lax monoidal transformation, i.e., it is appropriately unital and associative, see [EM53, Thm. 5.2] or [May67, 29.9]. The unit map is the unique chain map $\eta: \mathbb{Z}[0] \longrightarrow C(\mathbb{Z})$ which is the identity in dimension 0 .

The Alexander-Whitney map [ML63, VIII 8.5], [May67, 29.7]

$$
A W: C(A \otimes B) \longrightarrow C A \otimes C B
$$

goes in the direction opposite to the shuffle map; it is defined for a tensor product of $n$-simplices $a \in A_{n}$ and $b \in B_{n}$ by

$$
A W(a \otimes b)=\bigoplus_{p+q=n} \tilde{d}^{p} a \otimes d_{0}^{q} b .
$$

Algebraic 83 Geometric Topology, Volume 3 (2003) 
Here the 'front face' $\tilde{d}^{p}: A_{p+q} \longrightarrow A_{p}$ and the 'back face' $d_{0}^{q}: B_{p+q} \longrightarrow B_{q}$ are induced by the injective monotone maps $\tilde{\delta}^{p}:[p] \longrightarrow[p+q]$ and $\delta_{0}^{q}:[q] \longrightarrow$ $[p+q]$ defined by $\tilde{\delta}^{p}(i)=i$ and $\delta_{0}^{q}(i)=p+i$. The Alexander-Whitney map is a lax comonoidal transformation, (also referred to as an op-lax monoidal transformation) i.e., it is appropriately unital and associative.

Both the shuffle and the Alexander-Whitney map preserve the subcomplexes of degenerate simplices, compare [EM53, Lemma 5.3] or [May67, 29.8, 29.9]. Hence both maps factor over normalized chain complexes and induce maps

$$
\nabla: N A \otimes N B \longrightarrow N(A \otimes B) \text { and } A W: N(A \otimes B) \longrightarrow N A \otimes N B
$$

for which we use the same names. These restricted maps are again lax monoidal, respectively lax comonoidal, and the restricted unit maps are now isomorphisms $\mathbb{Z}[0] \cong N(\mathbb{Z})$.

Moreover, the composite map $A W \circ \nabla: C A \otimes C B \longrightarrow C A \otimes C B$ differs from the identity only by degenerate simplices. Hence on the level of normalized complexes, the composite

$$
N A \otimes N B \stackrel{\nabla}{\longrightarrow} N(A \otimes B) \stackrel{A W}{\longrightarrow} N A \otimes N B
$$

is the identity transformation. The composite of shuffle and Alexander-Whitney maps in the other order are naturally chain homotopic to the identity transformation. In particular, the shuffle map (2.4), the Alexander Whitney map (2.5) and their normalized versions (2.6) are all quasi-isomorphisms of chain complexes.

The shuffle map is also symmetric in the sense that for all simplicial abelian groups $A$ and $B$, the following square commutes

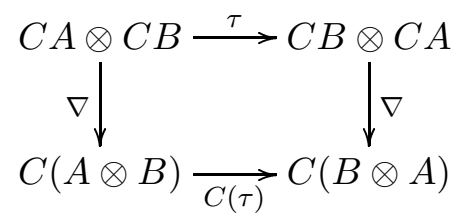

where $\tau$ denotes the symmetry isomorphism of the tensor products of either simplicial abelian groups or chain complexes. The normalized version (2.6) of the shuffle map is symmetric as well. However, the Alexander-Whitney map is not symmetric, nor is its normalized version.

We can turn the comonoidal structure on the normalization functor given by the Alexander-Whitney map (2.5) into a monoidal structure on the adjoint-inverse functor $\Gamma$ : we define

$$
\varphi_{C, D}: \Gamma C \otimes \Gamma D \longrightarrow \Gamma(C \otimes D)
$$


as the composite

$$
\begin{aligned}
\Gamma C \otimes \Gamma D \stackrel{\eta_{\Gamma C \otimes \Gamma D}}{\longrightarrow} \Gamma N(\Gamma C \otimes \Gamma D) \stackrel{\Gamma\left(A W_{\Gamma C, \Gamma D}\right)}{\longrightarrow} \\
\Gamma(N(\Gamma C) \otimes N(\Gamma D)) \stackrel{\Gamma\left(\epsilon_{C} \otimes \epsilon_{D}\right)}{\longrightarrow} \Gamma(C \otimes D) .
\end{aligned}
$$

The normalized Alexander-Whitney map (2.6) is surjective (it is split by the normalized shuffle map); since the unit and counit of the $(N, \Gamma)$-adjunction are isomorphisms, the monoidal map (2.8) is also a split surjection. The functor $\Gamma$ is not lax symmetric monoidal because the Alexander-Whitney map is not symmetric.

So $\Gamma$ induces a functor $\Gamma: \mathcal{D} G R \longrightarrow s \mathcal{R}$ on the associated categories of monoids: given a differential graded ring $R$ with product $\mu_{R}$ then $\Gamma R$ is a simplicial ring with product

$$
\Gamma R \otimes \Gamma R \stackrel{\varphi_{R, R}}{\longrightarrow} \Gamma(R \otimes R) \stackrel{\Gamma\left(\mu_{R}\right)}{\longrightarrow} \Gamma R .
$$

If we expand all the definitions, then the multiplication in $\Gamma R$ comes out as follows: the product of two $n$-simplices $x, y: N \Delta^{n} \longrightarrow R$ of $\Gamma R$ is the composition

$$
\begin{aligned}
N \Delta^{n} \stackrel{N(\text { diag })}{\longrightarrow} N\left(\Delta^{n} \times \Delta^{n}\right) \stackrel{A W_{\Delta^{n}, \Delta^{n}}}{\longrightarrow} \\
N \Delta^{n} \otimes N \Delta^{n} \stackrel{x \otimes y}{\longrightarrow} R \otimes R \stackrel{\mu_{R}}{\longrightarrow} R .
\end{aligned}
$$

Example 2.10 To give an idea of what the multiplication in $\Gamma R$ looks like, we calculate an explicit formula in the lowest dimension where something happens. The normalized chain complex of the simplicial 1-simplex $\Delta^{1}$ has as basis the cosets of the non-degenerate 1 -simplex $\iota \in \Delta_{1}^{1}$ and the two vertices $0=d_{1} \iota$ and $1=d_{0} \iota$; the differential in $N \Delta^{1}$ is determined by $d[\iota]=[1]-[0]$.

So for every 1-chain $r \in R_{1}$ of a differential graded ring $R$ we can define a chain map $\kappa r: N \Delta^{1} \longrightarrow R$ by setting

$$
(\kappa r)[\iota]=r,(\kappa r)[0]=0 \quad \text { and } \quad(\kappa r)[1]=d r .
$$

This defines a monomorphism $\kappa: R_{1} \longrightarrow c h^{+}\left(N \Delta^{1}, R\right)=(\Gamma R)_{1}$.

The composite map

$$
N \Delta^{1} \stackrel{N(\text { diag })}{\longrightarrow} N\left(\Delta^{1} \times \Delta^{1}\right) \stackrel{A W_{\Delta^{1}, \Delta^{1}}}{\longrightarrow} N \Delta^{1} \otimes N \Delta^{1}
$$

is given by

$$
\begin{aligned}
& {[\iota] \longmapsto[0] \otimes \iota \oplus \iota \otimes[1]} \\
& {[0] \longmapsto[0] \otimes[0] \text { and }[1] \longmapsto[1] \otimes[1] .}
\end{aligned}
$$


Hence we have

$$
(\kappa r \cdot \kappa s)[\iota]=(\kappa r)[0] \cdot(\kappa s)[\iota]+(\kappa r)[\iota] \cdot(\kappa s)[1]=r \cdot d s,
$$

and similarly $(\kappa r \cdot \kappa s)[0]=0$ and $(\kappa r \cdot \kappa s)[1]=d r \cdot d s$. In other words, we have shown the formula

$$
\kappa r \cdot \kappa s=\kappa(r \cdot d s)
$$

as 1-simplices of $\Gamma R$, for every pair of 1-chains $r, s \in R_{1}$. This formula already indicates that a simplicial ring of the form $\Gamma R$ is usually not commutative, even if $R$ is commutative in the graded sense.

\subsection{Monoidal properties of $\epsilon: N \Gamma \longrightarrow$ Id and $\eta:$ Id $\longrightarrow N \Gamma$}

The normalization functor is lax symmetric monoidal with structure map induced by the shuffle map (2.6). Thus, it also induces a functor on the categories of monoids $N: s \mathcal{R} \longrightarrow \mathcal{D} G R$. We shall see in the next proposition that $N$ is left inverse to $\Gamma$ on the level of rings; however, $N$ is not right inverse to $\Gamma$ on the point-set level, but only on the level of homotopy categories, see Remark 2.14 .

Lemma 2.11 The adjunction counit $\epsilon: N \Gamma \longrightarrow \mathrm{Id}_{c h^{+}}$is a monoidal transformation with respect to the composite monoidal structure on $N \Gamma$. More precisely, for every pair of connective chain complexes $C$ and $D$, the following diagram commutes

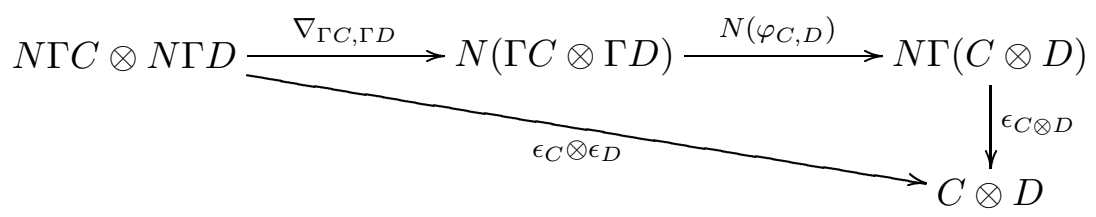

Proof The proof is a diagram chase, the main ingredient of which is the fact that the composite (2.7) of the normalized shuffle and Alexander-Whitney maps is the identity. We start with the identity

$$
\nabla_{\Gamma C, \Gamma D} \circ \epsilon_{N \Gamma C \otimes N \Gamma D}=\epsilon_{N(\Gamma C \otimes \Gamma D)} \circ N \Gamma\left(\nabla_{\Gamma C, \Gamma D}\right)
$$

as morphisms from $N \Gamma(N \Gamma C \otimes N \Gamma D)$ to $N(\Gamma C \otimes \Gamma D)$, which just says that $\epsilon$ is natural. The map $\epsilon_{N(\Gamma C \otimes \Gamma D)}$ is inverse to the map $N\left(\eta_{\Gamma C \otimes \Gamma D}\right): N(\Gamma C \otimes$ $\Gamma D) \longrightarrow N \Gamma N(\Gamma C \otimes \Gamma D)$, so we can rewrite the previous identity as

$$
N\left(\eta_{\Gamma C \otimes \Gamma D}\right) \circ \nabla_{\Gamma C, \Gamma D} \circ \epsilon_{N \Gamma C \otimes N \Gamma D}=N \Gamma\left(\nabla_{\Gamma C, \Gamma D}\right)
$$


as morphisms from $N \Gamma(N \Gamma C \otimes N \Gamma D)$ to $N \Gamma N(\Gamma C \otimes \Gamma D)$. Now we compose with the map $N \Gamma\left(A W_{\Gamma C, \Gamma D}\right): N \Gamma N(\Gamma C \otimes \Gamma D) \longrightarrow N \Gamma(N \Gamma C \otimes N \Gamma D)$ and exploit that the Alexander-Whitney map is left inverse to the shuffle map (see (2.7)); this yields

$$
\begin{aligned}
N \Gamma\left(A W_{\Gamma C, \Gamma D}\right) \circ N\left(\eta_{\Gamma C \otimes \Gamma D}\right) \circ \nabla_{\Gamma C, \Gamma D} \circ \epsilon_{N \Gamma C \otimes N \Gamma D} \\
=N \Gamma\left(A W_{\Gamma C, \Gamma D}\right) \circ N \Gamma\left(\nabla_{\Gamma C, \Gamma D}\right)=\operatorname{Id}_{N \Gamma(N \Gamma C \otimes N \Gamma D)} .
\end{aligned}
$$

Composing with $N \Gamma\left(\epsilon_{C} \otimes \epsilon_{D}\right): N \Gamma(N \Gamma C \otimes N \Gamma D) \longrightarrow N \Gamma(C \otimes D)$ and substituting the definition (2.8) of the monoidal transformation $\varphi$, we get

$$
\begin{aligned}
& N \Gamma\left(\epsilon_{C} \otimes \epsilon_{D}\right)= \\
& N \Gamma\left(\epsilon_{C} \otimes \epsilon_{D}\right) \circ N \Gamma\left(A W_{\Gamma C, \Gamma D}\right) \circ N\left(\eta_{\Gamma C \otimes \Gamma D}\right) \circ \nabla_{\Gamma C, \Gamma D} \circ \epsilon_{N \Gamma C \otimes N \Gamma D} \\
& =N\left(\varphi_{C, D}\right) \circ \nabla_{\Gamma C, \Gamma D} \circ \epsilon_{N \Gamma C \otimes N \Gamma D} .
\end{aligned}
$$

Since the counit $\epsilon$ is invertible, we can rewrite this as

$$
\epsilon_{C \otimes D} \circ N\left(\varphi_{C, D}\right) \circ \nabla_{\Gamma C, \Gamma D}=\epsilon_{C \otimes D} \circ N \Gamma\left(\epsilon_{C} \otimes \epsilon_{D}\right) \circ \epsilon_{N \Gamma C \otimes N \Gamma D}^{-1}=\epsilon_{C} \otimes \epsilon_{D}
$$

(the second equation is the naturality of $\epsilon$ ). This final identity is saying that the transformation $\epsilon: N \Gamma \longrightarrow$ Id is monoidal.

Proposition 2.13 The functor

$$
\Gamma: \mathcal{D} G R \longrightarrow s \mathcal{R}
$$

which sends a connective differential graded ring $R$ to the simplicial abelian group $\Gamma R$ with multiplication (2.9) is full and faithful. The composite endofunctor $N \Gamma$ of the category of differential graded rings is naturally isomorphic to the identity functor.

Proof The algebra valued functor $\Gamma$ is induced from an equivalence between the underlying categories of simplicial abelian groups and chain complexes. So in order to show that $\Gamma$ is fully faithful we have to prove that for every morphism $f: \Gamma R \longrightarrow \Gamma S$ of simplicial rings, the unique morphism $g: R \longrightarrow S$ of chain complexes which satisfies $f=\Gamma(g)$ is multiplicative and preserves the units.

The non-trivial part is to show that if $f: \Gamma R \longrightarrow \Gamma S$ is multiplicative, then the unique preimage $g: R \longrightarrow S$ is also multiplicative. Since $\Gamma$ is an equivalence on underlying categories, in order to show the relation $\mu_{S} \circ(g \otimes g)=g \circ \mu_{R}$ as chain maps from $R \otimes R$ to $S$, we may as well show the relation $\Gamma\left(\mu_{S} \circ(g \otimes g)\right)=$ 
$\Gamma\left(g \circ \mu_{R}\right)$ as maps of simplicial abelian groups from $\Gamma(R \otimes R)$ to $\Gamma S$. In the diagram

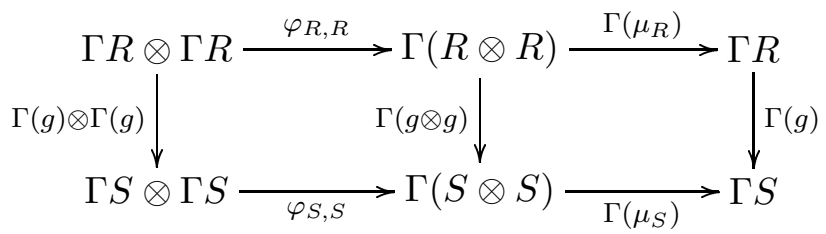

the left square commutes since $\varphi$ is natural, and the composite square commutes since $\Gamma(g)=f$ is multiplicative. Since the upper left morphism $\varphi_{R, R}$ is surjective, the right square commutes as well.

The natural isomorphism $N \Gamma \cong \mathrm{Id}$ is given by the counit $\epsilon: N \Gamma \longrightarrow \mathrm{Id}$ of the adjunction-equivalence between $N$ and $\Gamma$. This counit is an isomorphism, and it is monoidal by Lemma 2.11; this implies that on ring objects, the map $\epsilon$ is multiplicative; that $\epsilon$ is unital is even easier, so $\epsilon$ is a natural isomorphism of connective differential graded rings when evaluated on such objects.

Remark 2.14 The unit $\eta$ : Id $\longrightarrow \Gamma N$ of the adjunction-equivalence between $N$ and $\Gamma$ is not monoidal. More precisely, the composite

$$
\begin{aligned}
A \otimes B \stackrel{\eta_{A} \otimes \eta_{B}}{\longrightarrow} \Gamma(N A) \otimes \Gamma(N B) \stackrel{\varphi_{N A, N B}}{\longrightarrow} \\
\Gamma(N A \otimes N B) \stackrel{\Gamma\left(\nabla_{A, B}\right)}{\longrightarrow} \Gamma N(A \otimes B)
\end{aligned}
$$

need not in general be equal to the map $\eta_{A \otimes B}: A \otimes B \longrightarrow \Gamma N(A \otimes B)$. (Consider for example $A=B=\widetilde{\mathbb{Z}}(\Delta[1] / \partial \Delta[1])=\Gamma(\mathbb{Z}[1])$. In dimension one the composite $(2.15)$ is zero since it factors through $\Gamma(\mathbb{Z}[2])$. But in dimension one, $\eta_{A \otimes B}$ is an isomorphism between free abelian groups of rank two.) The situation is worse than for the counit $\epsilon$ (compare Lemma 2.11) because the composite of normalized shuffle and Alexander-Whitney map in the other order is only homotopic, but not equal to, the identity. Correspondingly, the composite (2.15) is homotopic, but not necessarily equal to, the map $\eta_{A \otimes B}$.

Nevertheless, the composite $\Gamma N$ is connected by a chain of two natural weak equivalences to the identity functor on the category of simplicial rings. In order to see this though, we have to refer to the Quillen equivalence of Theorem 1.1 (3). When considered as a ring valued functor, $N: s \mathcal{R} \longrightarrow \mathcal{D} G R$ has a left adjoint $L^{\text {mon }}: \mathcal{D} G R \longrightarrow s \mathcal{R}$, see Section 3.3 , which is not given by $\Gamma$ on underlying chain complexes. Moreover, the adjoint pair $N$ and $L^{\text {mon }}$ form a Quillen equivalence (Theorem $1.1(3)$ for $k=\mathbb{Z}$ ).

Given a simplicial ring $A$, we choose a cofibrant replacement

$$
q:(N A)^{c} \stackrel{\sim}{\longrightarrow} N A
$$


of $N A$ in the model category of connective differential graded rings (see Section 4.1). The model structure of differential graded rings is cofibrantly generated, so the small object argument provides a functorial choice of such a cofibrant replacement.

Since $N$ and $L^{\text {mon }}$ are a Quillen equivalence, the adjoint morphism

$$
\tilde{q}: L^{\operatorname{mon}}\left((N A)^{c}\right) \longrightarrow A
$$

is a weak equivalence of simplicial rings. By Lemma 2.11, the adjunction counit $\epsilon_{N A}: N \Gamma N A \longrightarrow N A$ is an isomorphism of differential graded rings. So we can form the composite multiplicative quasi-isomorphism $\epsilon_{N A}^{-1} \circ q:(N A)^{c} \longrightarrow$ $N \Gamma N A$ and take its monoid-valued adjoint

$$
L^{\operatorname{mon}}\left((N A)^{c}\right) \longrightarrow \Gamma N A \text {. }
$$

Since we have a Quillen equivalence, (2.16) is also a weak equivalence of simplicial rings. Altogether we obtain a chain of natural weak equivalences of simplicial rings

$$
\Gamma N A \stackrel{\sim}{\longleftarrow} L^{\mathrm{mon}}\left((N A)^{c}\right) \stackrel{\sim}{\longrightarrow} A .
$$

It is tempting to add the adjunction unit $\eta_{A}: \Gamma N A \longrightarrow A$ to directly connect the two simplicial rings; but $\eta_{A}$ is not in general a multiplicative map, and the resulting triangle involving $\Gamma N A, L^{\mathrm{mon}}\left((N A)^{c}\right)$ and $A$ need not commute!

\section{Weak monoidal equivalences}

In this section we first discuss the definitions of monoidal structures and their interactions with model category structures. Section 3.1 recalls the notion of a monoidal model category which is a model category with a compatible monoidal product. In Section 3.2 we define the notions of weak and strong monoidal Quillen equivalences between two monoidal model categories. A weak monoidal Quillen equivalence provides the basic properties necessary for lifting the Quillen equivalence to categories of monoids and modules. In a weak monoidal Quillen equivalence the right adjoint is assumed to be lax monoidal and hence induces functors on the associated categories of monoids and modules. This is not assumed for the left adjoint, though. Section 3.3 discusses the induced right adjoints and the relationship between the various context-dependent left adjoints. With this background we can then state our main result, Theorem 3.12, about Quillen equivalences on categories of monoids and modules. In Section 3.4 we discuss a criterion for establishing when a Quillen adjoint pair is a weak monoidal Quillen pair. The general criterion is given in Proposition 3.16; a variant for stable model categories appears in Proposition 3.17. 


\subsection{Monoidal model categories}

We consider a closed symmetric monoidal category [Bor94, 6.1] $\mathcal{C}$ and we denote the monoidal product by $\otimes$ (sometimes by $\wedge$ ), the unit object by $\mathbb{I}_{\mathcal{C}}$ and the internal function objects by $\operatorname{Hom}_{\mathcal{C}}(-,-)$. The internal function objects are almost never used explicitly (but see the proof of Proposition 3.16). But having a right adjoint makes sure that the monoidal product preserves colimits in both variables.

Definition 3.1 A model category $\mathcal{C}$ is a monoidal model category if it has a closed symmetric monoidal structure with product $\otimes$ and unit object $\mathbb{I}$ and satisfies the following two axioms.

Pushout product axiom Let $A \rightarrow B$ and $K \rightarrow L$ be cofibrations in $\mathcal{C}$. Then the map

$$
A \otimes L \amalg_{A \otimes K} B \otimes K \longrightarrow B \otimes L
$$

is also a cofibration. If in addition one of the former maps is a weak equivalence, so is the latter map.

Unit axiom Let $q: \mathbb{I}^{c} \stackrel{\sim}{\longrightarrow} \mathbb{I}$ be a cofibrant replacement of the unit object. Then for every cofibrant object $A$, the morphism $q \otimes \mathrm{Id}: \mathbb{I}^{c} \otimes A \longrightarrow \mathbb{I} \otimes A \cong A$ is a weak equivalence.

The previous definition is essentially the same as that of a symmetric monoidal model category in [Hov99, 4.2.6]; the only difference is that a model category in Hovey's sense is also equipped with a choice of cofibrant replacement functor, and Hovey requires the unit axiom for the particular functorial cofibrant replacement of the unit object. But given the pushout product axiom, then the unit axiom holds for one choice of cofibrant replacement if and only if it holds for any other choice. Of course the unit axiom is redundant if the unit object is cofibrant, as we often assume in this paper. The unit axiom did not occur in the definition of a monoidal model category in [SS00], since it did not play a role in the arguments of that paper.

In this paper we are interested in model categories of monoids [ML71, VII 3] and modules (i.e., objects with an action by a monoid, see [ML71, VII 4]) in some underlying monoidal model category. In the cases we study the model structure is always transferred or lifted from the underlying category to the category of more structured objects as in the following definition.

Definition 3.2 Consider a functor $R: \mathcal{T} \longrightarrow \mathcal{C}$ to a model category $\mathcal{C}$ with a left adjoint $L: \mathcal{C} \longrightarrow \mathcal{T}$. We call an object of $\mathcal{T}$ a cell object if it can be 
obtained from the initial object as a (possibly transfinite) composition [Hov99, 2.1.1] of pushouts along morphisms of the form $L f$, for $f$ a cofibration in $\mathcal{C}$. We say that the functor $R$ creates a model structure if

- the category $\mathcal{T}$ supports a model structure (necessarily unique) in which a morphism $f: X \longrightarrow Y$ is a weak equivalence, respectively fibration, if and only the morphism $R f$ is a weak equivalence, respectively fibration, in $\mathcal{C}$, and

- every cofibrant object in $\mathcal{T}$ is a retract of a cell object.

The typical example of Definition 3.2 occurs when the model structure on $\mathcal{C}$ is cofibrantly generated ([SS00, 2.2] or [Hov99, 2.1.17]) and then a lifting theorem is used to lift the model structure to $\mathcal{T}$ along the adjoint functor pair. In [SS00, 4.1] we give sufficient conditions for ensuring that the forgetful functors to an underlying monoidal model category $\mathcal{C}$ create model structures for monoids or modules and algebras: it suffices that the model structure on $\mathcal{C}$ is cofibrantly generated, that the objects of $\mathcal{C}$ are small relative to the whole category ([SS00, $\S 2]$ or [Hov99, 2.1.3]) and that the monoid axiom [SS00, 3.3] holds.

Another situation where the forgetful functor creates a model structure for the category of monoids is when all objects in $\mathcal{C}$ are fibrant and when there exists an 'interval with coassociative, comultiplication'; for more details compare the example involving chain complexes [SS00, 2.3 (2), Sec. 5] or more generally [BM, Prop. 4.1].

\subsection{Monoidal Quillen pairs}

The main goal for this paper is to give conditions which show that a Quillen equivalence between two monoidal model categories induces a Quillen equivalence on the categories of monoids, modules and algebras. For this we have to assume that the functors involved preserve the monoidal structure in some way. We assume that the right adjoint is lax monoidal in the sense of the following definition.

Definition 3.3 A lax monoidal functor between monoidal categories is a functor $R: \mathcal{C} \longrightarrow \mathcal{D}$ equipped with a morphism $\nu: \mathbb{I}_{\mathcal{D}} \longrightarrow R\left(\mathbb{I}_{\mathcal{C}}\right)$ and natural morphisms

$$
\varphi_{X, Y}: R X \wedge R Y \longrightarrow R(X \otimes Y)
$$

which are coherently associative and unital (see diagrams 6.27 and 6.28 of [Bor94]). A lax monoidal functor is strong monoidal if the morphisms $\nu$ and $\varphi_{X, Y}$ are isomorphisms.

Algebraic 83 Geometric Topology, Volume 3 (2003) 
Consider a lax monoidal functor $R: \mathcal{C} \longrightarrow \mathcal{D}$ between monoidal categories, with monoidal structure maps $\nu$ and $\varphi_{X, Y}$. If $R$ has a left adjoint $\lambda: \mathcal{D} \longrightarrow \mathcal{C}$, we can consider the adjoint $\tilde{\nu}: \lambda\left(\mathbb{I}_{\mathcal{D}}\right) \longrightarrow \mathbb{I}_{\mathcal{C}}$ of $\nu$ and the natural map

$$
\widetilde{\varphi}: \lambda(A \wedge B) \longrightarrow \lambda A \otimes \lambda B
$$

adjoint to the composite

$$
A \wedge B \stackrel{\eta_{A} \wedge \eta_{B}}{\longrightarrow} R \lambda A \wedge R \lambda B \stackrel{\varphi_{\lambda A, \lambda B}}{\longrightarrow} R(\lambda A \otimes \lambda B) .
$$

The map $\widetilde{\varphi}$ can equivalently be defined as the composition

$$
\begin{aligned}
\lambda(A \wedge B) \stackrel{\lambda\left(\eta_{A} \wedge \eta_{B}\right)}{\longrightarrow} \lambda(R \lambda A & \wedge R \lambda B) \stackrel{\lambda\left(\varphi_{\lambda_{A}, \lambda_{B}}\right)}{\longrightarrow} \\
\lambda & \lambda(\lambda A \otimes \lambda B) \stackrel{\epsilon_{\lambda_{A} \otimes \lambda_{B}}}{\longrightarrow} \lambda A \otimes \lambda B ;
\end{aligned}
$$

here $\eta$ and $\epsilon$ denote the unit respectively counit of the adjunction. With respect to these maps, $\lambda$ is a lax comonoidal functor (also referred to as an op-lax monoidal functor). The map $\widetilde{\varphi}$ need not be an isomorphism; in that case $\lambda$ does not have a monoidal structure, and so it does not pass to a functor on the monoid and module categories.

Definition 3.6 A pair of adjoint functors

$$
\mathcal{D} \underset{\lambda}{\stackrel{R}{\leftrightarrows}} \mathcal{C}
$$

between model categories is a Quillen adjoint pair if the right adjoint $R$ preserves fibrations and trivial fibrations. A Quillen adjoint pair induces adjoint total derived functors between the homotopy categories by [Qui67, I.4.5]. A Quillen functor pair is a Quillen equivalence if the total derived functors are adjoint equivalences of the homotopy categories.

A weak monoidal Quillen pair between monoidal model categories $\mathcal{C}$ and $\mathcal{D}$ consists of a Quillen adjoint functor pair $(\lambda: \mathcal{D} \longleftrightarrow \mathcal{C}: R)$ with a lax monoidal structure on the right adjoint

$$
\varphi_{X, Y}: R X \wedge R Y \longrightarrow R(X \otimes Y), \quad \nu: \mathbb{I}_{\mathcal{D}} \longrightarrow R\left(\mathbb{I}_{\mathcal{C}}\right)
$$

such that the following two conditions hold:

(i) for all cofibrant objects $A$ and $B$ in $\mathcal{D}$ the comonoidal map (3.4)

$$
\widetilde{\varphi}: \lambda(A \wedge B) \longrightarrow \lambda A \otimes \lambda B
$$

is a weak equivalence in $\mathcal{C}$ and 
(ii) for some (hence any) cofibrant replacement $q: \mathbb{I}_{\mathcal{D}}^{c} \stackrel{\sim}{\longrightarrow} \mathbb{I}_{\mathcal{D}}$ of the unit object in $\mathcal{D}$, the composite map

$$
\lambda\left(\mathbb{I}_{\mathcal{D}}^{c}\right) \stackrel{\lambda(q)}{\longrightarrow} \lambda\left(\mathbb{I}_{\mathcal{D}}\right) \stackrel{\tilde{\nu}}{\longrightarrow} \mathbb{I}_{\mathcal{C}}
$$

is a weak equivalence in $\mathcal{C}$.

A strong monoidal Quillen pair is a weak monoidal Quillen pair for which the comonoidal maps $\widetilde{\varphi}$ and $\tilde{\nu}$ are isomorphisms. Note that if $\mathbb{I}_{\mathcal{D}}$ is cofibrant and $\lambda$ is strong monoidal, then $R$ is lax monoidal and the Quillen pair is a strong monoidal Quillen pair.

A weak (respectively strong) monoidal Quillen pair is a weak monoidal Quillen equivalence (respectively strong monoidal Quillen equivalence) if the underlying Quillen pair is a Quillen equivalence.

Strong monoidal Quillen pairs are the same as monoidal Quillen adjunctions in the sense of Hovey [Hov99, 4.2.16]. The weak monoidal Quillen pairs do not occur in Hovey's book.

\subsection{Various left adjoints}

As any lax monoidal functor, the right adjoint $R: \mathcal{C} \longrightarrow \mathcal{D}$ of a weak monoidal Quillen pair induces various functors on the categories of monoids and modules. More precisely, for a monoid $A$ in $\mathcal{C}$ with multiplication $\mu: A \otimes A \longrightarrow A$ and unit $\eta: \mathbb{I}_{\mathcal{C}} \longrightarrow A$, the monoid structure on $R A$ is given by the composite maps

$$
R A \wedge R A \stackrel{\varphi_{A, A}}{\longrightarrow} R(A \otimes A) \stackrel{R(\mu)}{\longrightarrow} R A \quad \text { and } \quad \mathbb{I}_{\mathcal{D}} \stackrel{\nu}{\longrightarrow} R\left(\mathbb{I}_{\mathcal{C}}\right) \stackrel{R(\eta)}{\longrightarrow} R A .
$$

Similarly, for an $A$-module $M$ with action morphism $\alpha: M \otimes A \longrightarrow M$, the $\mathcal{D}$-object $R M$ becomes an $R A$-module via the composite morphism

$$
R M \wedge R A \stackrel{\varphi_{M, A}}{\longrightarrow} R(M \otimes A) \stackrel{R(\alpha)}{\longrightarrow} R M .
$$

In our context, $R$ has a left adjoint $\lambda: \mathcal{D} \longrightarrow \mathcal{C}$. The left adjoint inherits an 'adjoint' comonoidal structure $\widetilde{\varphi}: \lambda(A \wedge B) \longrightarrow \lambda A \otimes \lambda B$ and $\tilde{\nu}: \lambda\left(\mathbb{I}_{\mathcal{D}}\right) \longrightarrow \mathbb{I}_{\mathcal{C}}$, see (3.4), and the pair is strong monoidal if $\widetilde{\varphi}$ and $\tilde{\nu}$ are isomorphisms. In that case, the left adjoint becomes a strong monoidal functor via the inverses

$$
\widetilde{\varphi}^{-1}: \lambda A \otimes \lambda B \longrightarrow \lambda(A \wedge B) \quad \text { and } \quad \tilde{\nu}^{-1}: \mathbb{I}_{\mathcal{C}} \longrightarrow \lambda\left(\mathbb{I}_{\mathcal{D}}\right) \text {. }
$$

Via these maps, $\lambda$ then lifts to a functor on monoids and modules, and the lift is again adjoint to the module- or algebra-valued version of $R$. 
However, we want to treat the more general situation of weak monoidal Quillen pairs. In that case, the functors induced by $R$ on modules and algebras still have left adjoints. However, on underlying $\mathcal{D}$-objects, these left adjoints are not usually given by the original left adjoint $\lambda$. As far as $R$ is concerned, we allow ourselves the abuse of notation to use the same symbol for the original lax monoidal functor $R$ from $\mathcal{C}$ to $\mathcal{D}$ as well as for its structured versions. However, for the left adjoints it seems more appropriate to use different symbols, which we now introduce.

In our applications we always assume that the forgetful functor from $\mathcal{C}$-monoids to $\mathcal{C}$ creates a model structure. In particular, the category of $\mathcal{C}$-algebras has colimits and the forgetful functor has a left adjoint 'free monoid' (or 'tensor algebra') functor [ML71, VII 3, Thm. 2]

$$
T_{\mathcal{C}} X=\coprod_{n \geq 0} X^{\otimes n}=\mathbb{I}_{\mathcal{C}} \amalg X \amalg(X \otimes X) \amalg \ldots,
$$

with multiplication given by juxtaposition, and similarly for $T_{\mathcal{D}}$. This implies that the monoid-valued lift $R: \mathcal{C}$-Monoid $\longrightarrow \mathcal{D}$-Monoid again has a left adjoint

$$
L^{\text {mon }}: \mathcal{D} \text {-Monoid } \longrightarrow \mathcal{C} \text {-Monoid }
$$

Indeed, for a $\mathcal{D}$-monoid $B$, the value of the left adjoint can be defined as the coequalizer of the two $\mathcal{C}$-monoid morphisms

$$
T_{\mathcal{C}}\left(\lambda\left(T_{\mathcal{D}} B\right)\right) \Longrightarrow T_{\mathcal{C}}(\lambda B) \longrightarrow L^{\text {mon }} B
$$

(where the forgetful functors are not displayed). One of the two maps is obtained from the adjunction unit $T_{\mathcal{D}} B \longrightarrow B$ by applying the the composite functor $T_{\mathcal{C}} \lambda$; the other map is the unique $\mathcal{C}$-monoid morphism which restricts to the $\mathcal{C}$-morphism

$$
\lambda\left(T_{\mathcal{D}} B\right) \cong \coprod_{n \geq 0} \lambda\left(B^{\wedge n}\right) \stackrel{\amalg \widetilde{\varphi}}{\longrightarrow} \coprod_{n \geq 0}(\lambda B)^{\otimes n} \cong T_{\mathcal{C}}(\lambda B) .
$$

Since $R$ preserves the underlying objects, the monoid left adjoint and the original left adjoint are related via a natural isomorphism of functors from $\mathcal{D}$ to monoids in $\mathcal{C}$

$$
L^{\text {mon }} \circ T_{\mathcal{D}} \cong T_{\mathcal{C}} \circ \lambda .
$$

As in the above case of monoids, the module valued functor $R: \operatorname{Mod}-A \longrightarrow$ Mod-RA has a left adjoint

$$
L^{A}: \operatorname{Mod}-R A \longrightarrow \operatorname{Mod}-A
$$


as soon as free $R$-modules and coequalizers of $R$-modules exist. Since $R$ preserves the underlying objects, this module left adjoint and the original left adjoint are related via a natural isomorphism of functors from $\mathcal{D}$ to $A$-modules

$$
L^{A} \circ(-\wedge R A) \cong(-\otimes A) \circ \lambda
$$

here $X \otimes A$ is the free $A$-module generated by a $\mathcal{C}$-object $X$, and similarly $Y \wedge R A$ for a $\mathcal{D}$-object $Y$.

Finally, for a monoid $B$ in $\mathcal{D}$, the lax monoidal functor $R$ induces a functor from the category of $L^{\text {mon }} B$-modules to the category of $B$-modules; this is really the composite functor

$$
\operatorname{Mod}-\left(L^{\mathrm{mon}} B\right) \stackrel{R}{\longrightarrow} \operatorname{Mod}-R\left(L^{\mathrm{mon}} B\right) \stackrel{\eta^{*}}{\longrightarrow} \operatorname{Mod}-B
$$

where the second functor is restriction of scalars along the monoid homomorphism (the adjunction unit) $\eta: B \longrightarrow R\left(L^{\text {mon }} B\right)$. We denote by

$$
L_{B}: \operatorname{Mod}-B \longrightarrow \operatorname{Mod}-\left(L^{\mathrm{mon}} B\right)
$$

the left adjoint to the functor $R: \mathcal{M} o d-\left(L^{\text {mon }} B\right) \longrightarrow \mathcal{M}$ od- $B$. This left adjoint factors as a composition

$$
\operatorname{Mod}-B \stackrel{-\otimes_{B} R\left(L^{\mathrm{mon}} B\right)}{\longrightarrow} \operatorname{Mod}-R\left(L^{\mathrm{mon}} B\right) \stackrel{L^{L^{\mathrm{mon}} B}}{\longrightarrow} \operatorname{Mod}-\left(L^{\mathrm{mon}} B\right)
$$

(the first functor is extension of scalars along $B \longrightarrow R\left(L^{\text {mon }} B\right)$ ); the left adjoint is related to the free module functors by a natural isomorphism

$$
L_{B} \circ(-\wedge B) \cong\left(-\otimes L^{\mathrm{mon}} B\right) \circ \lambda .
$$

We repeat that if the monoidal pair is strong monoidal, then left adjoints $L^{\text {mon }}$ and $L_{B}$ are given by the original left adjoint $\lambda$, which is then monoidal via the inverse of $\widetilde{\varphi}$. Moreover, the left adjoint $L^{A}: \operatorname{Mod}-R A \longrightarrow \operatorname{Mod}-A$ is then given by the formula

$$
L^{A}(M)=\lambda(M) \wedge_{\lambda(R A)} A,
$$

where $A$ is a $\lambda(R A)$-module via the adjunction counit $\epsilon: \lambda(R A) \longrightarrow A$. In general however, $\lambda$ does not pass to monoids and modules, and the difference between $\lambda$ and the structured adjoints $L^{\text {mon }}, L^{A}$ and $L_{B}$ is investigated in Proposition 5.1 below.

We need just one more definition before stating our main theorem.

Definition 3.11 Let $\left(\mathcal{C}, \otimes, \mathbb{I}_{\mathcal{C}}\right)$ be a monoidal model category such that the forgetful functors create model structures for modules over any monoid. We say 
that Quillen invariance holds for $\mathcal{C}$ if for every weak equivalence of $\mathcal{C}$-monoids $f: R \longrightarrow S$, restriction and extension of scalars along $f$ induce a Quillen equivalence between the respective module categories.

$$
-\wedge_{R} S: \operatorname{Mod}-(R) \longleftrightarrow \operatorname{Mod}-S: f^{*}
$$

A sufficient condition for Quillen invariance in $\mathcal{C}$ is that for every cofibrant right $R$-module $M$ the functor $M \otimes_{R}$ - takes weak equivalences of left $R$-modules to weak equivalences in $\mathcal{C}$ (see for example [SS00, 4.3] or Theorem 6.1 (2)).

Theorem 3.12 Let $R: \mathcal{C} \longrightarrow \mathcal{D}$ be the right adjoint of a weak monoidal Quillen equivalence. Suppose that the unit objects in $\mathcal{C}$ and $\mathcal{D}$ are cofibrant.

(1) Consider a cofibrant monoid $B$ in $\mathcal{D}$ such that the forgetful functors create model structures for modules over $B$ and modules over $L^{\text {mon }} B$. Then the adjoint functor pair

$$
L_{B}: \operatorname{Mod}-B \longleftrightarrow \mathcal{M o d}-\left(L^{\mathrm{mon}} B\right): R
$$

is a Quillen equivalence.

(2) Suppose that Quillen invariance holds in $\mathcal{C}$ and $\mathcal{D}$. Then for any fibrant monoid $A$ in $\mathcal{C}$ such that the forgetful functors create model structures for modules over $A$ and modules over $R A$, the adjoint functor pair

$$
L^{A}: \operatorname{Mod}-R A \longleftrightarrow \operatorname{Mod}-A: R
$$

is a Quillen equivalence. If the right adjoint $R$ preserves weak equivalences between monoids and the forgetful functors create model structures for modules over any monoid, then this holds for any monoid $A$ in $\mathcal{C}$.

(3) If the forgetful functors create model structures for monoids in $\mathcal{C}$ and $\mathcal{D}$, then the adjoint functor pair

$$
L^{\text {mon }}: \mathcal{D} \text {-Monoid } \leftrightarrows \mathcal{C} \text {-Monoid }: R
$$

is a Quillen equivalence between the model categories of monoids.

The statements (1) and (2) for modules in the previous Theorem 3.12 generalize to 'rings with many objects' or enriched categories, see Theorem 6.5. The proof of Theorem 3.12 appears in Section 5. 


\subsection{A Criterion for weak monoidal pairs}

In this section we assume that $\lambda$ and $R$ form a Quillen adjoint functor pair between two monoidal model categories $\left(\mathcal{C}, \otimes, \mathbb{I}_{\mathcal{C}}\right)$ and $\left(\mathcal{D}, \wedge, \mathbb{I}_{\mathcal{D}}\right)$. We establish a sufficient condition for when the Quillen pair is a weak monoidal Quillen pair: if the unit object $\mathbb{I}_{\mathcal{D}}$ detects weak equivalences (see Definition 3.14), then the lax comonoidal transformation (3.4)

$$
\widetilde{\varphi}: \lambda(A \wedge B) \longrightarrow \lambda A \otimes \lambda B .
$$

is a weak equivalence on cofibrant objects.

To define what it means to detect weak equivalences, we use the notion of a cosimplicial resolution which was introduced by Dwyer and Kan [DK80, 4.3] as a device to provide homotopy meaningful mapping spaces. More recently, (co-)simplicial resolutions have been called (co-)simplicial frames [Hov99, 5.2.7]. Cosimplicial objects in any model category admit the Reedy model structure in which the weak equivalences are the cosimplicial maps which are levelwise weak equivalences and the cofibrations are the Reedy cofibrations; the latter are a special class of levelwise cofibrations defined with the use of 'latching objects'. The Reedy fibrations are defined by the right lifting property for Reedy trivial cofibrations or equivalently with the use of matching objects; see [Hov99, 5.2.5] for details on the Reedy model structure. A cosimplicial resolution of an object $A$ of $\mathcal{C}$ is a cofibrant replacement $A^{*} \longrightarrow c A$ in the Reedy model structure of the constant cosimplicial object $c A$ with value $A$. In other words, a cosimplicial resolution is a Reedy cofibrant cosimplicial object which is homotopically constant in the sense that each cosimplicial structure map is a weak equivalence in $\mathcal{C}$. Cosimplicial resolutions always exist $[\mathrm{DK} 80,4.5]$ and are unique up to level equivalence under $A$.

If $A^{*}$ is a cosimplicial object and $Y$ is an object of $\mathcal{C}$, then there is a simplicial set $\operatorname{map}\left(A^{*}, Y\right)$ of $\mathcal{C}$-morphisms defined by

$$
\operatorname{map}\left(A^{*}, Y\right)_{n}=\mathcal{C}\left(A^{n}, Y\right) .
$$

If $f: A^{*} \longrightarrow B^{*}$ is a level equivalence between Reedy cofibrant cosimplicial objects and $Y$ is a fibrant object, then the induced map of mapping spaces $\operatorname{map}(f, Y): \operatorname{map}\left(B^{*}, Y\right) \longrightarrow \operatorname{map}\left(A^{*}, Y\right)$ is a weak equivalence [Hov99, 5.4.8]. Hence the homotopy type of the simplicial set $\operatorname{map}\left(A^{*}, Y\right)$, for $A^{*}$ a cosimplicial resolution, depends only on the underlying object $A^{0}$ of $\mathcal{C}$. The path components of the simplicial set $\operatorname{map}\left(A^{*}, Y\right)$ are in natural bijection with the set of homotopy classes of maps from $A^{0}$ to $Y$ [Hov99, 5.4.9],

$$
\pi_{0} \operatorname{map}\left(A^{*}, Y\right) \cong\left[A^{0}, Y\right]_{\mathrm{Ho}(\mathcal{C})} .
$$


Remark 3.13 The notion of a cosimplicial resolution is modeled on the 'product with simplices'. More precisely, in a simplicial model category $\mathcal{C}$, we have a pairing between objects of $\mathcal{C}$ and simplicial sets. So if we let $n$ vary in the simplicial category $\Delta$, we get a cosimplicial object $\Delta^{n} \times A$ associated to every object $A$ of $\mathcal{C}$. If $A$ is cofibrant, then this cosimplicial object is Reedy cofibrant and homotopically constant, i.e., a functorial cosimplicial resolution of $A$. Moreover, the $n$-simplices of the simplicial set $\operatorname{map}(A, Y)-$ which is also part of the simplicial structure - are in natural bijection with the set of morphisms from $\Delta^{n} \times A$ to $Y$,

$$
\operatorname{map}(A, Y)_{n}=\mathcal{C}\left(\Delta^{n} \times A, Y\right) .
$$

So the mapping spaces with respect to the preferred resolution $\Delta^{*} \times A$ coincide with the usual simplicial function spaces [Qui67, II.1.3].

Definition 3.14 An object $A$ of a model category $\mathcal{C}$ detects weak equivalences if for some (hence any) cosimplicial frame $A^{*}$ of $A$ the following condition holds: a morphism $f: Y \longrightarrow Z$ between fibrant objects is a weak equivalence if and only if the map

$$
\operatorname{map}\left(A^{*}, f\right): \operatorname{map}\left(A^{*}, Y\right) \longrightarrow \operatorname{map}\left(A^{*}, Z\right)
$$

is a weak equivalence of simplicial sets.

Example 3.15 Every one point space detects weak equivalences in the model category of topological spaces with respect to weak homotopy equivalences. The one point simplicial set detects weak equivalences of simplicial sets with respect to weak homotopy equivalences. For a simplicial ring $R$, the free $R$-module of rank one detects weak equivalences of simplicial $R$-modules.

Let $A$ be a connective differential graded algebra. Then the free differential graded $A$-module of rank one detects weak equivalences of connective $A$ modules. Indeed, a cosimplicial resolution of the free $A$-module of rank one is given by $N \Delta^{*} \otimes A$, i.e., by tensoring $A$ with the normalized chain complexes of the standard simplices. With respect to this resolution the mapping space into another connective $A$-module $Y$ has the form

$$
\operatorname{map}_{\mathcal{M o d}-A}\left(N \Delta^{*} \otimes A, Y\right) \cong \operatorname{map}_{c h^{+}}\left(N \Delta^{*}, Y\right)=\Gamma Y .
$$

A homomorphism of connective $A$-modules is a quasi-isomorphism if and only if it becomes a weak equivalences of simplicial sets after applying the functor $\Gamma$. Hence the free $A$-module of rank one detects weak equivalences, as claimed.

Now we formulate the precise criterion for a Quillen functor pair to be weakly monoidal. The proof of this proposition is in Section 5 . 
Proposition 3.16 Consider a Quillen functor pair $(\lambda: \mathcal{D} \longleftrightarrow \mathcal{C}: R)$ between monoidal model categories together with a lax monoidal structure on the right adjoint $R$. Suppose further that

(1) for some (hence any) cofibrant replacement $q: \mathbb{I}_{\mathcal{D}}^{c} \longrightarrow \mathbb{I}_{\mathcal{D}}$ of the unit object in $\mathcal{D}$, the composite map

$$
\lambda\left(\mathbb{I}_{\mathcal{D}}^{c}\right) \stackrel{\lambda(q)}{\longrightarrow} \lambda\left(\mathbb{I}_{\mathcal{D}}\right) \stackrel{\tilde{\nu}}{\longrightarrow} \mathbb{I}_{\mathcal{C}}
$$

is a weak equivalence in $\mathcal{C}$, where the second map is adjoint to the monoidal structure map $\nu: \mathbb{I}_{\mathcal{D}} \longrightarrow R\left(\mathbb{I}_{\mathcal{C}}\right)$;

(2) the unit object $\mathbb{I}_{\mathcal{D}}$ detects weak equivalences in $\mathcal{D}$.

Then $R$ and $\lambda$ are a weak monoidal Quillen pair.

This criterion works well in unstable situations such as (non-negatively graded) chain complexes and simplicial abelian groups. For the stable case, though, this notion of detecting weak equivalences is often too strong. Thus, we say an object $A$ of a stable model category $\mathcal{C}$ stably detects weak equivalences if $f: Y \longrightarrow Z$ is a weak equivalence if and only if $[A, Y]_{*}^{\mathrm{Ho} \mathcal{C}} \longrightarrow[A, Z]_{*}^{\mathrm{Ho} \mathcal{C}}$ is an isomorphism of the $\mathbb{Z}$-graded abelian groups of morphisms in the triangulated homotopy category $\mathrm{Ho}_{\mathrm{C}}$. For example, a (weak) generator stably detects weak equivalences by [SS03, 2.2.1]. The unstable notion above would correspond to just considering $* \geq 0$.

Modifying the proof of Proposition 3.16 by using the graded morphisms in the homotopy category instead of the mapping spaces introduced above proves the following stable criterion.

Proposition 3.17 Consider a Quillen functor pair $(\lambda: \mathcal{D} \leftrightarrows \mathcal{C}: R)$ between monoidal stable model categories together with a lax monoidal structure on the right adjoint $R$. Suppose further that

(1) for some (hence any) cofibrant replacement $q: \mathbb{I}_{\mathcal{D}}^{c} \longrightarrow \mathbb{I}_{\mathcal{D}}$ of the unit object in $\mathcal{D}$, the composite map

$$
\lambda\left(\mathbb{I}_{\mathcal{D}}^{c}\right) \stackrel{\lambda(q)}{\longrightarrow} \lambda\left(\mathbb{I}_{\mathcal{D}}\right) \stackrel{\tilde{\nu}}{\longrightarrow} \mathbb{I}_{\mathcal{C}}
$$

is a weak equivalence in $\mathcal{C}$, where the second map is adjoint to the monoidal structure map $\nu: \mathbb{I}_{\mathcal{D}} \longrightarrow R\left(\mathbb{I}_{\mathcal{C}}\right)$;

(2) the unit object $\mathbb{I}_{\mathcal{D}}$ stably detects weak equivalences in $\mathcal{D}$.

Then $R$ and $\lambda$ are a weak monoidal Quillen pair. 


\section{Chain complexes and simplicial abelian groups, re- visited}

Dold and Kan showed that the category of non-negatively graded chain complexes is equivalent to the category of simplicial abelian groups, see for example [Do58, Thm. 1.9]. The equivalence is given by the normalization functor $N: s \mathcal{A} b \longrightarrow c h^{+}$and its inverse $\Gamma: c h^{+} \longrightarrow s \mathcal{A} b$. Because the two functors are inverses to each other, they are also adjoint to each other on both sides. Hence the normalized chain complex $N$ and its inverse functor $\Gamma$ give rise to two different weak monoidal Quillen equivalences. Each choice of right adjoint comes with a particular monoidal transformation, namely the shuffle map (for $N$ as the right adjoint, see Section 4.2) or the Alexander-Whitney map (for $\Gamma$ as the right adjoint, see Section 4.3).

In Section 4.1 we recall the supporting model category structures on chain complexes, simplicial abelian groups and the associated categories of monoids and modules. In Section 4.2, we show that Theorem 3.12 parts (2) and (3) imply Theorem 1.1 parts (2) and (3) respectively. In Section 4.3, we show that Theorem 3.12 part (2) implies Theorem 1.1 part (1). In Section 4.4 we then use the fact that the shuffle map for $N$ is lax symmetric monoidal to deduce Theorem 1.1 part (4) from Theorem 3.12 part (3).

\subsection{Model structures}

Let $k$ be a commutative ring. The category $c h_{k}^{+}$of non-negatively graded chain complexes of $k$-modules supports the projective model structure: weak equivalences are the quasi-isomorphisms, fibrations are the chain maps which are surjective in positive dimensions, and cofibrations are the monomorphisms with dimensionwise projective cokernel. More details can be found in [DS95, Sec. 7] or [Hov99, 2.3.11, 4.2.13] (the references in [Hov99] actually treat $\mathbb{Z}$ graded chain complexes, but the arguments for $c h^{+}$are similar; there is also an injective model structure for $\mathbb{Z}$-graded chain complexes with the same weak equivalences, but we do not use this model structure).

The model category structure on simplicial $k$-modules has as weak equivalences and fibrations the weak homotopy equivalences and Kan fibrations on underlying simplicial sets; the cofibrations are the retracts of free maps in the sense of [Qui67, II.4.11 Rem. 4]. For more details see [Qui67, II.4, II.6].

The functor $N$ is an inverse equivalence of categories, and it exactly matches the notions of cofibrations, fibrations and weak equivalences in the two above 
model structures. So $N$ and its inverse $\Gamma$ can be viewed as Quillen equivalences in two ways, with either functor playing the role of the left or the right adjoint.

Both model structures are compatible with the tensor products of Section 2.2 in the sense that they satisfy the pushout product axiom (compare [Hov99, 4.2.13]). A model category on simplicial rings with fibrations and weak equivalences determined on the underlying simplicial abelian groups (which in turn are determined by the underlying simplicial sets) was established by Quillen in [Qui67, II.4, Theorem 4]. Similarly, there is a model structure on differential graded rings with weak equivalences the quasi-isomorphisms and fibrations the maps which are surjective in positive degrees; see [Jar97]. These model category structures also follow from verifying the monoid axiom [SS00, Def. 3.3] and using Theorem 4.1 of [SS00]; see also [SS00, Section 5].

\subsection{A first weak monoidal Quillen equivalence}

Let $k$ be a commutative ring. We view the normalization functor

$$
N: s \mathcal{M o d}-k \longrightarrow c h_{k}^{+}
$$

from simplicial $k$-modules to non-negatively graded chain complexes of $k$ modules as the right adjoint and its inverse $\Gamma(2.2)$ as the left adjoint.

We consider $N$ as a lax monoidal functor via the shuffle map (2.6). The shuffle map $\nabla: N A \otimes N B \longrightarrow N(A \otimes B)$ is a chain homotopy equivalence for every pair of simplicial $k$-modules, cofibrant or not, with homotopy inverse the Alexander-Whitney map [May67, 29.10]. Since $\Gamma$ takes quasi-isomorphisms to weak equivalences of simplicial $k$-modules, and since the unit and counit of the adjunction between $N$ and $\Gamma$ are isomorphisms, the description (3.4) of the comonoidal transformation for the left adjoint shows that the map

$$
\widetilde{\nabla}_{C, D}: \Gamma(C \otimes D) \longrightarrow \Gamma C \otimes \Gamma D
$$

is a weak equivalence of simplicial $k$-modules for all connective complexes of $k$-modules.

In other words, with respect to the shuffle map, $N$ is the right adjoint of a weak monoidal Quillen equivalence between simplicial $k$-modules and connective chain complexes of $k$-modules. Since the unit objects are cofibrant, we can apply Theorem 3.12. Part (3) shows that normalization is the right adjoint of a Quillen equivalence from simplicial $k$-algebras to connective differential graded $k$-algebras; this proves part (3) of Theorem 1.1. Quillen invariance holds for simplicial rings, and normalization preserves all weak equivalences; 
so for $k=\mathbb{Z}$, part (2) of Theorem 3.12 shows that for every simplicial ring $A$, normalization is the right adjoint of a Quillen equivalence from simplicial $A$-modules to connective differential graded $N A$-modules; this proves part (2) of Theorem 1.1.

All the above does not use the fact that the shuffle map for $N$ is symmetric monoidal. We explore the consequences of this in 4.4 .

\subsection{Another weak monoidal Quillen equivalence}

Again, let $k$ be a commutative ring. This time we treat the normalization functor $N: s \mathcal{M}$ od- $k \longrightarrow c h_{k}^{+}$as the left adjoint and its inverse functor $\Gamma: c h_{k}^{+} \longrightarrow$ $s \mathcal{M o d}-k$ as right adjoint to $N$. The monoidal structure on $\Gamma$ defined in (2.8) is made so that the comonoidal transformation (3.4) for the left adjoint $N$ is precisely the Alexander-Whitney map

$$
A W: N(A \otimes B) \longrightarrow N A \otimes N B .
$$

The Alexander-Whitney map is a chain homotopy equivalence for arbitrary simplicial $k$-modules $A$ and $B$, with homotopy inverse the shuffle map [May67, 29.10]; hence $\Gamma$ becomes the right adjoint of a weak monoidal Quillen equivalence.

Since the unit objects are cofibrant, we can again apply Theorem 3.12. Part (2) shows that for every connective differential graded ring $R$, the functor $\Gamma$ is the right adjoint of a Quillen equivalence from connective differential graded $R$-modules to simplicial $\Gamma R$-modules; this proves part (1) of Theorem 1.1.

\subsection{Modules and algebras over a commutative simplicial ring}

The shuffle map (2.4) is lax symmetric monoidal, and so is its extension

$$
\nabla: N A \otimes N B \longrightarrow N(A \otimes B)
$$

to normalized chain complexes. In sharp contrast to this, the AlexanderWhitney map is not symmetric. This has the following consequences:

- If $A$ is a commutative simplicial ring, then the normalized chains $N A$ form a differential graded algebra which is commutative in the graded sense, i.e., we have

$$
x y=(-1)^{|x||y|} y x
$$

for homogeneous elements $x$ and $y$ in $N A$. 
- The functor $N$ inherits a lax monoidal structure when considered as a functor from simplicial $A$-modules (with tensor product over $A$ ) to connective differential graded $N A$-modules (with tensor product over $N A$ ). More precisely, there is a unique natural chain map

$$
\nabla^{A}: N M \otimes_{N A} N\left(M^{\prime}\right) \longrightarrow N\left(M \otimes_{A} M^{\prime}\right) .
$$

for $A$-modules $M$ and $M^{\prime}$, such that the square

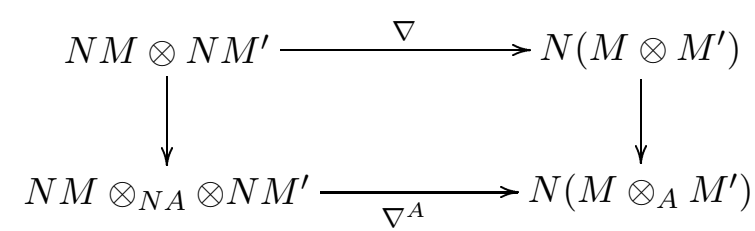

commutes (where the vertical morphisms are the natural quotient maps). This much does not depend on commutativity of the product of $A$. However, if $A$ is commutative, then $\nabla^{A}$ constitutes a lax symmetric monoidal functor from $A$ modules to $N A$-modules; this uses implicitly that the monoidal transformation $\nabla$ is symmetric, hence compatible with the isomorphism of categories between left and right modules over $A$ and $N A$.

Now let $L^{A}: \operatorname{Mod}-N A \longrightarrow \mathcal{M}$ od- $A$ denote the left adjoint of $N$ when viewed as a functor from left $A$-modules to left modules over $N A$ (compare Section 3.3). Then the lax comonoidal map for $L^{A}$ has the form

$$
L^{A}\left(W \otimes_{N A} W^{\prime}\right) \longrightarrow L^{A}(W) \otimes_{A} L^{A}\left(W^{\prime}\right)
$$

for a pair of left $N A$-modules $W$ and $W^{\prime}$. We claim that this map is a weak equivalence for cofibrant modules $W$ and $W^{\prime}$ by appealing to Theorem 3.16. Indeed, the unit objects of the two tensor products are the respective free modules of rank one, which are cofibrant. The unit map $\eta: L^{A}(N A) \longrightarrow A$ is even an isomorphism, and the free $N A$-module of rank one detects weak equivalences by Example 3.15. So Theorem 3.16 applies to show that the adjoint functor pair

$$
\operatorname{Mod}-N A \underset{L^{A}}{\stackrel{N}{\leftrightarrows}} \operatorname{Mod}-A
$$

is a weak monoidal Quillen pair. These two adjoint functors form a Quillen equivalence by part (2) of Theorem 3.12.

Now we can apply part (3) of Theorem 3.12. The conclusion is that the normalized chain complex functor is the right adjoint of a Quillen equivalence from the model category of simplicial $A$-algebras to the model category of connective differential graded algebras over the commutative differential graded ring $N A$. In other words, this proves part (4) of Theorem 1.1 


\section{Proofs}

This section contains the proofs of the main results of the paper, namely Theorem 3.12 and the criterion for being weakly monoidal, Proposition 3.16.

\subsection{Proof of Theorem 3.12}

This proof depends on a comparison of different kinds of left adjoints in Proposition 5.1. For this part we assume that $\lambda$ and $R$ form a weak monoidal Quillen pair, in the sense of Definition 3.6, between two monoidal model categories $\left(\mathcal{C}, \otimes, \mathbb{I}_{\mathcal{C}}\right)$ and $\left(\mathcal{D}, \wedge, \mathbb{I}_{\mathcal{D}}\right)$. As before, the lax monoidal transformation of $R$ is denoted $\varphi_{X, Y}: R X \wedge R Y \longrightarrow R(X \otimes Y)$; it is 'adjoint' to the comonoidal transformation (3.4)

$$
\widetilde{\varphi}: \lambda(A \wedge B) \longrightarrow \lambda A \otimes \lambda B .
$$

This comonoidal map need not be an isomorphism; in that case $\lambda$ does not have a monoidal structure, and so it does not pass to a functor between monoid and module categories. However, part of the definition of a weak monoidal Quillen pair is that $\widetilde{\varphi}$ is a weak equivalence whenever $A$ and $B$ are cofibrant.

In our applications, the monoidal functor $R$ has left adjoints on the level of monoids and modules (see Section 3.3), and the following proposition compares these 'structured' left adjoints to the underlying left adjoint $\lambda$. If the monoidal transformation $\widetilde{\varphi}$ of (3.4) happens to be an isomorphism, then so are the comparison morphisms $\chi_{B}$ and $\chi_{W}$ which occur in the following proposition; so for strong monoidal Quillen pairs the following proposition has no content.

In Definition 3.2 we defined the notion of a 'cell object' relative to a functor to a model category. In the following proposition, the notions of cell object are taken relative to the forgetful functors from algebras, respectively modules, to the underlying monoidal model category.

Proposition 5.1 Let $(\lambda: \mathcal{D} \leftrightarrows \mathcal{C}: R)$ be a weak monoidal Quillen pair between monoidal model categories with cofibrant unit objects.

(1) Suppose that the functor $R: \mathcal{C}$-Monoid $\longrightarrow \mathcal{D}$-Monoid has a left adjoint $L^{\text {mon }}$. Then for every cell monoid $B$ in $\mathcal{D}$, the $\mathcal{C}$-morphism

$$
\chi_{B}: \lambda B \longrightarrow L^{\mathrm{mon}} B
$$

which is adjoint to the underlying $\mathcal{D}$-morphism of the adjunction unit $B \longrightarrow R\left(L^{\text {mon }} B\right)$ is a weak equivalence. 
(2) Let $B$ be a cell monoid in $\mathcal{D}$ for which the functor $R: \mathcal{M o d}-\left(L^{\text {mon }} B\right) \longrightarrow$ $\mathcal{M}$ od- $B$ has a left adjoint $L_{B}$. Then for every cell $B$-module $M$, the $\mathcal{C}$ morphism

$$
\chi_{M}: \lambda M \longrightarrow L_{B} M
$$

which is adjoint to the underlying $\mathcal{D}$-morphism of the adjunction unit $M \longrightarrow R\left(L_{B} M\right)$ is a weak equivalence.

Proof Part (1): The left adjoint $L^{\text {mon }}$ takes the initial $\mathcal{D}$-monoid $\mathbb{I}_{\mathcal{D}}$ to the initial $\mathcal{C}$-monoid $\mathbb{I}_{\mathcal{C}}$ and the map $\chi_{\mathbb{I}_{\mathcal{D}}}: \lambda\left(\mathbb{I}_{\mathcal{D}}\right) \longrightarrow L^{\text {mon }} \mathbb{I}_{\mathcal{D}} \cong \mathbb{I}_{\mathcal{C}}$ is the adjoint $\tilde{\nu}$ of the unit map $\nu: \mathbb{I}_{\mathcal{D}} \longrightarrow R\left(\mathbb{I}_{\mathcal{C}}\right)$. By definition of a weak monoidal Quillen pair, $\tilde{\nu}$ is a weak equivalence. Hence $\chi$ is a weak equivalence for the initial $\mathcal{D}$-monoid $\mathbb{I}_{\mathcal{D}}$.

Now we proceed by a cell induction argument, along free extensions of cofibrations in $\mathcal{D}$. We assume that $\chi_{B}$ is a weak equivalence for some cell $\mathcal{D}$-monoid $B$. Since the unit $\mathbb{I}_{\mathcal{D}}$ is cofibrant, $B$ is also cofibrant in the underlying category $\mathcal{D}$ by an inductive application of [SS00, Lemma 6.2].

We consider another monoid $P$ obtained from $B$ by a single 'cell attachment', i.e., a pushout in the category of $\mathcal{D}$-monoids of the form

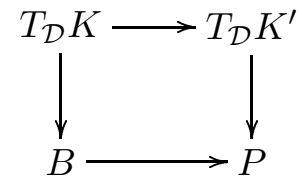

where $K \longrightarrow K^{\prime}$ is a cofibration in $\mathcal{D}$. We may assume without loss of generality that $K$ and $K^{\prime}$ are in fact cofibrant. Indeed, $P$ is also a pushout of the diagram

$$
B \longleftarrow{ }^{\epsilon} T_{\mathcal{D}} B \longrightarrow T_{\mathcal{D}}\left(B \amalg_{K} K^{\prime}\right)
$$

where $\epsilon: T_{\mathcal{D}} B \longrightarrow B$ is the counit of the free monoid adjunction and $B \amalg_{K} K^{\prime}$ denotes the pushout in the underlying category $\mathcal{D}$. Since $B$ is cofibrant in $\mathcal{D}$, the morphism $B \longrightarrow B \amalg_{K} K^{\prime}$ is a cofibration between cofibrant objects in $\mathcal{D}$, and it can be used instead of the original cofibration $K \longrightarrow K^{\prime}$.

Free extensions of monoids are analyzed in the proof of [SS00, 6.2] and we make use of that description. The underlying object of $P$ can be written as a colimit of a sequence of cofibrations $P_{n-1} \longrightarrow P_{n}$ in $\mathcal{D}$, with $P_{0}=B$ such that each morphism $P_{n-1} \longrightarrow P_{n}$ is a pushout in $\mathcal{D}$ of a particular cofibration $Q_{n}\left(K, K^{\prime}, B\right) \longrightarrow\left(B \wedge K^{\prime}\right)^{\wedge n} \wedge B$. Since $\lambda$ is a left adjoint on these underlying categories, $\lambda$ applied to each corner of these pushouts is still a pushout square. 
Since $L^{\text {mon }}$ is a left adjoint on the categories of monoids, it preserves pushouts of monoids. So we have the following pushout of $\mathcal{C}$-monoids

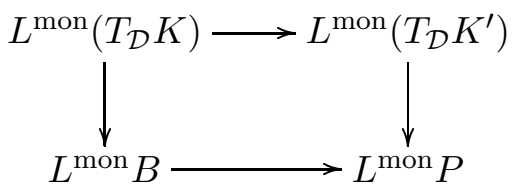

Because of the natural isomorphism (3.7) between $L^{\text {mon }}\left(T_{\mathcal{D}} K\right)$ and $T_{\mathcal{C}}(\lambda K)$, the pushout $L^{\text {mon }} P$ is thus the free extension of $L^{\text {mon }} B$ along the cofibration $\lambda K \longrightarrow \lambda K^{\prime}$ between cofibrant objects in $\mathcal{C}$. In particular, $L^{\text {mon }} P$ is the colimit in $\mathcal{C}$ of a sequence of cofibrations $R_{n-1} \longrightarrow R_{n}$ each of which is a pushout in $\mathcal{C}$ of a cofibration $Q_{n}\left(\lambda K, \lambda K^{\prime}, L^{\text {mon }} B\right) \longrightarrow\left(L^{\text {mon }} B \wedge \lambda K^{\prime}\right)^{\wedge n} \wedge L^{\text {mon }} B$.

The map $\chi_{P}: \lambda P \longrightarrow L^{\text {mon }} P$ preserves these filtrations: it takes $\lambda P_{n}$ to $R_{n}$. We now show by induction that for each $n$ the map $\lambda P_{n} \longrightarrow R_{n}$ is a weak equivalence in $\mathcal{C}$. We show that the map on each of the other three corners of the pushout squares defining $\lambda P_{n}$ and $R_{n}$ is a weak equivalence between cofibrant objects; then we apply [Hov99, 5.2.6] to conclude that the map of pushouts is also a weak equivalence. By induction we assume $\lambda P_{n-1} \longrightarrow R_{n-1}$ is a weak equivalence. A second corner factors as

$$
\begin{aligned}
\lambda\left(\left(B \wedge K^{\prime}\right)^{\wedge n} \wedge B\right) \stackrel{\widetilde{\varphi}}{\longrightarrow} \quad\left(\lambda B \wedge \lambda K^{\prime}\right)^{\wedge n} \wedge \lambda B \\
\stackrel{\left(\chi_{B} \wedge \mathrm{Id}\right)^{\wedge n} \wedge \chi_{B}}{\longrightarrow}\left(L^{\mathrm{mon}} B \wedge \lambda K^{\prime}\right)^{\wedge n} \wedge L^{\mathrm{mon}} B .
\end{aligned}
$$

Since the first map is an (iterated) instance of the comonoidal transformation $\widetilde{\varphi}$, and since $B$ is cofibrant in the underlying category $\mathcal{D}$, the first map is a weak equivalence by hypothesis. Since $L^{\text {mon }} B$ is a cell monoid in $\mathcal{C}$ and the unit object $\mathbb{I}_{\mathcal{C}}$ is cofibrant, $L^{\text {mon }} B$ is cofibrant in the underlying category $\mathcal{C}$ by [SS00, Lemma 6.2]. By induction we know that $\chi_{B}: \lambda B \longrightarrow L^{\mathrm{mon}} B$ is a weak equivalence; since all objects in sight are cofibrant and smashing with a cofibrant object preserves weak equivalences between cofibrant objects, the second map is also a weak equivalence.

The third corner works similarly: the map factors as

$$
\lambda Q_{n}\left(K, K^{\prime}, B\right) \longrightarrow Q_{n}\left(\lambda K, \lambda K^{\prime}, \lambda B\right) \longrightarrow Q_{n}\left(\lambda K, \lambda K^{\prime}, L^{\text {mon }} B\right) .
$$

Here $Q_{n}$ itself is constructed as a pushout of an $n$-cube where each map is a cofibration between cofibrant objects. Using a variant of [Hov99, 5.2.6] for $n$ cubes, the hypothesis on the comonoidal transformation $\widetilde{\varphi}$ and induction shows that this third corner is also a weak equivalence.

Since each filtration map is a cofibration between cofibrant objects and $\lambda P_{n} \longrightarrow$ $R_{n}$ is a weak equivalence for each $n$, we can apply [Hov99, 1.1.12, 5.1.5] to 
conclude that the map of colimits $\lambda P \longrightarrow L^{\text {mon }} P$ is a weak equivalence since it is the colimit of a weak equivalence between cofibrant objects in the Reedy model category of directed diagrams. Similarly, for the transfinite compositions allowed in building up a cell object, [Hov99, 1.1.12, 5.1.5] shows that the map of colimits is a weak equivalence.

(2) We use a similar induction for cell $B$-modules. Again at transfinite composition steps, [Hov99, 1.1.12, 5.1.5] gives the necessary conclusion; so we are left with considering the single cell attachments. Suppose the statement has been verified for some cell $B$-module $M$. Since $B$ is a cell monoid, it is cofibrant in the underlying category $\mathcal{D}$, and so is $M$, by induction on the 'number of cells'. Suppose $M^{\prime}$ is obtained from $M$ as a free extension of $B$-modules, i.e, it is a pushout of a diagram

$$
M \longleftarrow K \wedge B \longrightarrow K^{\prime} \wedge B
$$

Where $K \longrightarrow K^{\prime}$ is a cofibration in $\mathcal{D}$. By the same trick as in the first part, we can assume without loss of generality that $K$ and $K^{\prime}$ are cofibrant in $\mathcal{D}$; this exploits the fact that $B$ is also cofibrant in $\mathcal{D}$.

Since $L_{B}$ is a left adjoint, it preserves pushouts. On the other hand, the forgetful functor from $B$-modules to $\mathcal{D}$ and $\lambda: \mathcal{C} \longrightarrow \mathcal{D}$ also preserve pushouts, so we get a commutative diagram in $\mathcal{C}$

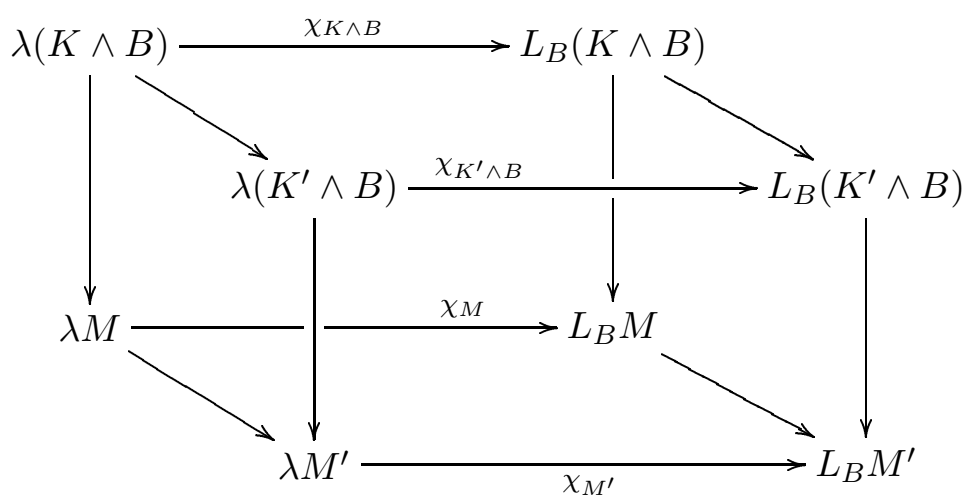

in which the left and right faces are pushout squares. Because of the natural isomorphisms $L_{B}(K \wedge B) \cong(\lambda A) \otimes L^{\text {mon }} B$ and $L_{B}\left(K^{\prime} \wedge B\right) \cong \lambda K^{\prime} \otimes L^{\text {mon }} B$ of (3.10), the pushout $L_{B} M^{\prime}$ is thus the free extension of $L_{B} M$ along the cofibration $\lambda K \longrightarrow \lambda K^{\prime}$ between cofibrant objects in $\mathcal{C}$.

By assumption, the map $\chi_{M}$ is a weak equivalence. The map $\chi_{K \wedge B}$ factors as the composite

$$
\lambda(K \wedge B) \stackrel{\widetilde{\varphi}}{\longrightarrow} \lambda K \otimes \lambda B \stackrel{\operatorname{Id} \otimes \chi_{B}}{\longrightarrow} \lambda K \otimes L^{\mathrm{mon}} B .
$$


Since $B$ is cofibrant in the underlying category $\mathcal{D}$ and the first map is an instance of the comonoidal transformation $\widetilde{\varphi}$, it is a weak equivalence. Since the map $\lambda B \longrightarrow L^{\mathrm{mon}} B$ is a weak equivalence by Part (1) and all objects in sight are cofibrant, the second map of (5.2) is also a weak equivalence. Hence $\chi_{K \wedge B}$ is a weak equivalence, and similarly for $\chi_{K^{\prime} \wedge B}$. Since the maps on each of the three initial corners of the pushout squares defining $\lambda M^{\prime}$ and $L_{B} M^{\prime}$ are weak equivalence between cofibrant objects, the map of pushouts is also a weak equivalence (see for example [Hov99, 5.2.6]). So $\chi_{M^{\prime}}: \lambda M^{\prime} \longrightarrow L_{B} M^{\prime}$ is a weak equivalence.

Proof of Theorem 3.12 Since the fibrations and trivial fibrations of monoids and modules are defined on the underlying category, the right adjoint $R$ is a right Quillen functor in all cases.

(3) Consider a cofibrant $\mathcal{D}$-monoid $B$ and a fibrant $\mathcal{C}$-monoid $Y$. We have to show that a monoid homomorphism $B \longrightarrow R Y$ is a weak equivalence if and only if its adjoint $L^{\text {mon }} B \longrightarrow Y$ is a weak equivalence. In the underlying category $\mathcal{C}$, we can consider the composite

$$
\lambda B \stackrel{\chi_{B}}{\longrightarrow} L^{\text {mon }} B \longrightarrow Y
$$

which is adjoint to the underlying $\mathcal{D}$-morphism of $B \longrightarrow R Y$.

Since the forgetful functor creates (in the sense of Definition 3.2) the model structure in the category of $\mathcal{D}$-monoids, every cofibrant $\mathcal{D}$-monoid $B$ is a retract of a cell monoid by definition. So the morphism $\chi_{B}: \lambda B \longrightarrow L^{\text {mon }} B$ is a weak equivalence by Proposition 5.1 (1). Since $\mathbb{I}_{\mathcal{D}}$ is cofibrant, a cofibrant $\mathcal{D}$-monoid is also cofibrant as an object of $\mathcal{D}$ by [SS00,6.2]. Thus, since $\lambda$ and $R$ form a Quillen equivalence on the underlying categories, the composite map (5.3) is a weak equivalence if and only if $B \longrightarrow R Y$ is a weak equivalence. So $L^{\text {mon }}$ and $R$ form a Quillen equivalence between the categories of monoids in $\mathcal{C}$ and $\mathcal{D}$.

(1) This is very similar to part (3), but using the second part of Proposition 5.1 instead of the first part.

(2) Let $\psi: A \longrightarrow A^{\mathrm{f}}$ be a fibrant replacement in the category of $\mathcal{C}$-monoids. If $A$ is already fibrant, take $A^{\mathrm{f}}=A$, otherwise assume $R$ preserves all weak equivalences, so either way $R \psi: R A \longrightarrow R\left(A^{\mathrm{f}}\right)$ is a weak equivalence of $\mathcal{D}$ monoids. Let $\kappa: R\left(A^{\mathrm{f}}\right)^{c} \longrightarrow R\left(A^{\mathrm{f}}\right)$ be a cofibrant replacement in the category of $\mathcal{D}$-monoids. Let $\widetilde{\kappa}: L^{\operatorname{mon}}\left(R\left(A^{\mathrm{f}}\right)^{c}\right) \longrightarrow A^{\mathrm{f}}$ be its adjoint; by part (3), this adjoint $\widetilde{\kappa}$ is a weak equivalence of monoids in $\mathcal{C}$. We have a commutative 
diagram of right Quillen functors

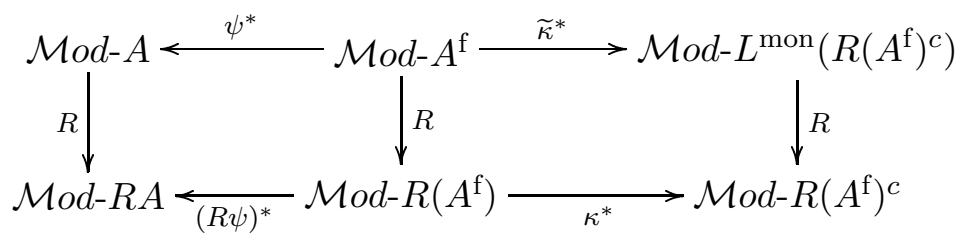

in which the horizontal functors are restrictions of scalars along the various weak equivalences of monoids; these are right Quillen equivalences by Quillen invariance. By part (1) the right vertical functor is a right Quillen equivalence. Hence, the middle and left vertical functors are also right Quillen equivalences.

\subsection{Proof of Proposition 3.16}

We are given a Quillen functor pair $(R: \mathcal{C} \leftrightarrows \mathcal{D}: \lambda)$ between monoidal model categories together with a lax monoidal structure on the right adjoint $R$. Moreover, the unit object $\mathbb{I}_{\mathcal{D}}$ detects weak equivalences in $\mathcal{D}$ (in the sense of Definition 3.14) and for some (hence any) cofibrant replacement $\mathbb{I}_{\mathcal{D}}^{c} \longrightarrow \mathbb{I}_{\mathcal{D}}$ of the unit object in $\mathcal{D}$, the composite map $\lambda\left(\mathbb{I}_{\mathcal{D}}^{c}\right) \longrightarrow \lambda\left(\mathbb{I}_{\mathcal{D}}\right) \stackrel{\tilde{\nu}}{\rightarrow} \mathbb{I}_{\mathcal{C}}$ is a weak equivalence in $\mathcal{C}$. We have to show that $R$ and $\lambda$ are a weak monoidal Quillen pair.

Our first step is to show that for every cofibrant object $B$ of $\mathcal{D}$ and every fibrant object $Y$ of $\mathcal{C}$, a certain map

$$
R \operatorname{Hom}_{\mathcal{C}}(\lambda B, Y) \longrightarrow \operatorname{Hom}_{\mathcal{D}}(B, R Y)
$$

is a weak equivalence in $\mathcal{D}$. Here $\operatorname{Hom}_{\mathcal{C}}(-,-)$ and $\operatorname{Hom}_{\mathcal{D}}(-,-)$ denote the internal function objects in $\mathcal{C}$ respectively $\mathcal{D}$, which are part of the closed symmetric monoidal structures. The map (5.4) is adjoint to the composition

$$
\begin{aligned}
R \operatorname{Hom}_{\mathcal{C}}(\lambda B, Y) \wedge B \stackrel{\mathrm{Id} \wedge \eta}{\longrightarrow} R \operatorname{Hom}_{\mathcal{C}}(\lambda B, Y) \wedge R(\lambda B) \\
\stackrel{\varphi}{\longrightarrow} R\left(\operatorname{Hom}_{\mathcal{C}}(\lambda B, Y) \otimes \lambda B\right) \stackrel{R(\text { eval })}{\longrightarrow} R Y .
\end{aligned}
$$

Choose a cosimplicial resolution $\mathbb{I}_{\mathcal{D}}^{*}$ of the unit object; in particular, in cosimplicial dimension zero we get a cofibrant replacement $\mathbb{I}_{\mathcal{D}}^{0} \stackrel{\sim}{\longrightarrow} \mathbb{I}_{\mathcal{D}}$ of the unit object. Since $\mathbb{I}_{\mathcal{D}}$ detects weak equivalences in $\mathcal{D}$ and the objects $R \operatorname{Hom}_{\mathcal{C}}(\lambda B, Y)$ and $\operatorname{Hom}_{\mathcal{D}}(B, R Y)$ are fibrant, we can prove that (5.4) is a weak equivalence by showing that we get a weak equivalence of mapping spaces

$$
\operatorname{map}_{\mathcal{D}}\left(\mathbb{I}_{\mathcal{D}}^{*}, R \operatorname{Hom}_{\mathcal{C}}(\lambda B, Y)\right) \longrightarrow \operatorname{map}_{\mathcal{D}}\left(\mathbb{I}_{\mathcal{D}}^{*}, \operatorname{Hom}_{\mathcal{D}}(B, R Y)\right)
$$


The adjunction isomorphisms between $R$ and $\lambda$ and between the monoidal products and internal function objects allow us to rewrite this map as

$$
\operatorname{map}_{\mathcal{C}}(\widetilde{\varphi}, Y): \operatorname{map}_{\mathcal{C}}\left(\lambda\left(\mathbb{I}_{\mathcal{D}}^{*}\right) \otimes \lambda B, Y\right) \longrightarrow \operatorname{map}_{\mathcal{C}}\left(\lambda\left(\mathbb{I}_{\mathcal{D}}^{*} \wedge B\right), Y\right),
$$

where the cosimplicial map

$$
\widetilde{\varphi}: \lambda\left(\mathbb{I}_{\mathcal{D}}^{*} \wedge B\right) \longrightarrow \lambda\left(\mathbb{I}_{\mathcal{D}}^{*}\right) \otimes \lambda B
$$

is an instance of the comonoidal map $\widetilde{\varphi}$ in each cosimplicial dimension. We consider the commutative diagram in $\mathcal{C}$

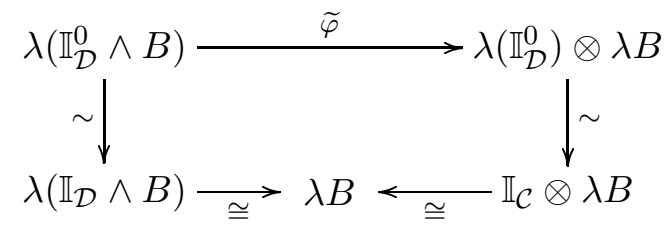

whose top horizontal map is the component of (5.5) in cosimplicial dimension zero. Since $\mathbb{I}_{\mathcal{D}}^{0}$ is a cofibrant replacement of the unit in $\mathcal{D}$, the map $\mathbb{I}_{\mathcal{D}}^{0} \wedge B \longrightarrow$ $\mathbb{I}_{\mathcal{D}} \wedge B \cong B$ is a weak equivalence between cofibrant objects, and so the left vertical map is a weak equivalence. By hypothesis (1), the composite map $\lambda\left(\mathbb{I}_{\mathcal{D}}^{0}\right) \longrightarrow \lambda\left(\mathbb{I}_{\mathcal{D}}\right) \longrightarrow \mathbb{I}_{\mathcal{C}}$ is a cofibrant replacement of the unit in $\mathcal{C}$, so smashing it with the cofibrant object $\lambda B$ gives the right vertical weak equivalence.

We conclude that (5.5) is a weak equivalence in dimension zero; every left Quillen functor such as $\lambda,-\wedge B$ or $-\otimes \lambda B$ preserves cosimplicial resolutions, so (5.5) is a level equivalence between Reedy cofibrant objects. So the induced map on mapping spaces $\operatorname{map}(-, Y)$ is a weak equivalence by [Hov99, 5.4.8], and so the map (5.4) is a weak equivalence in $\mathcal{D}$.

Now we play the game backwards. If $A$ is another cofibrant object of $\mathcal{D}$, then $\mathbb{I}_{\mathcal{D}}^{*} \wedge A$ is a cosimplicial resolution whose dimension zero object $\mathbb{I}_{\mathcal{D}}^{0} \wedge A$ is weakly equivalent to $A$. Since the map (5.4) is a weak equivalence between fibrant objects and the functor $\operatorname{map}_{\mathcal{D}}\left(\mathbb{I}_{\mathcal{D}}^{*} \wedge A,-\right)$ is a right Quillen functor [Hov99, 5.4.8], we get an induced weak equivalence on mapping spaces

$$
\operatorname{map}_{\mathcal{D}}\left(\mathbb{I}_{\mathcal{D}}^{*} \wedge A, R \operatorname{Hom}_{\mathcal{C}}(\lambda B, Y)\right) \longrightarrow \operatorname{map}_{\mathcal{D}}\left(\mathbb{I}_{\mathcal{D}}^{*} \wedge A, \operatorname{Hom}_{\mathcal{D}}(B, R Y)\right) .
$$

Using adjunction isomorphisms again, this map can be rewritten as

$$
\operatorname{map}_{\mathcal{C}}(\widetilde{\varphi}, Y): \operatorname{map}_{\mathcal{C}}\left(\lambda\left(\mathbb{I}_{\mathcal{D}}^{*} \wedge A\right) \otimes \lambda B, Y\right) \longrightarrow \operatorname{map}_{\mathcal{C}}\left(\lambda\left(\mathbb{I}_{\mathcal{D}}^{*} \wedge A \wedge B\right), Y\right) \text {. }
$$

where

$$
\widetilde{\varphi}: \lambda\left(\mathbb{I}_{\mathcal{D}}^{*} \wedge A \wedge B\right) \longrightarrow \lambda\left(\mathbb{I}_{\mathcal{D}}^{*} \wedge A\right) \otimes \lambda B
$$

is another instance of the comonoidal map $\widetilde{\varphi}$ in each cosimplicial dimension. 
Since $\lambda\left(\mathbb{I}_{\mathcal{D}}^{*} \wedge A\right) \otimes \lambda B$ is a cosimplicial frame of the cofibrant object $\lambda A \otimes \lambda B$, the components of the mapping $\operatorname{space}^{\operatorname{map}_{\mathcal{C}}}\left(\lambda\left(\mathbb{I}_{\mathcal{D}}^{*} \wedge A\right) \otimes \lambda B, Y\right)$ are in natural bijection with the morphisms from $\lambda A \otimes \lambda B$ in the homotopy category of $\mathcal{C}$; similarly the components of $\operatorname{map}_{\mathcal{C}}\left(\lambda\left(\mathbb{I}_{\mathcal{D}}^{*} \wedge A \wedge B\right), Y\right)$ are isomorphic to $[\lambda(A \wedge$ $B), Y]_{\mathrm{Ho}(\mathcal{C})}$. So we conclude that the comonoidal map $\widetilde{\varphi}: \lambda(A \wedge B) \longrightarrow \lambda A \otimes \lambda B$ induces a bijection of homotopy classes of maps into every fibrant object $Y$. Thus the map $\widetilde{\varphi}$ is a weak equivalence in $\mathcal{C}$, and so we indeed have a weak monoidal Quillen pair.

\section{Some enriched model category theory}

In this section we develop some general theory of modules over 'rings with several objects' based on a monoidal model category. Most of this is a relatively straightforward generalization from the case of 'ring objects' or monoids to 'rings with several objects'. Throughout this section, $\mathcal{C}$ is a monoidal model category (Definition 3.1) with product $\otimes$ and unit object $\mathbb{I}$. For the special case where $\mathcal{C}$ is the category of symmetric spectra, this material can also be found in [SS03, Sec. A.1]. Additional material on the homotopy theory of $\mathcal{C}$-categories can be found in [Du01].

\subsection{Modules over $\mathcal{C}$-categories}

Let $I$ be any set and let $\mathcal{O}$ be a $\mathcal{C} I$-category [Bor94, 6.2], i.e., a category enriched over $\mathcal{C}$ whose set of objects is $I$. This means that for all $i, j \in I$ there is a morphism object $\mathcal{O}(i, j) \in \mathcal{C}$, unit morphisms $\mathbb{I} \longrightarrow \mathcal{O}(i, i)$ and coherently associative and unital composition morphisms

$$
\mathcal{O}(j, k) \otimes \mathcal{O}(i, j) \longrightarrow \mathcal{O}(i, k) \text {. }
$$

One may think of $\mathcal{O}$ as a 'ring/monoid with many objects', indexed by $I$, enriched in $\mathcal{C}$. Indeed if $I=\{*\}$ has only one element, then $\mathcal{O}$ is completely determined by the endomorphism $\mathcal{C}$-monoid $\mathcal{O}(*, *)$. Moreover the $\mathcal{O}$-modules as defined below coincide with the $\mathcal{O}(*, *)$-modules in the ordinary sense.

A (right) $\mathcal{O}$-module is a contravariant $\mathcal{C}$-functor [Bor94, 6.2.3] from $\mathcal{O}$ to the category $\mathcal{C}$; explicitly, an $\mathcal{O}$-module $M$ consists of $\mathcal{C}$-objects $M(i)$ for $i \in I$ and $\mathcal{C}$-morphisms

$$
M(j) \otimes \mathcal{O}(i, j) \longrightarrow M(i)
$$

which are appropriately associative and unital. A map of $\mathcal{O}$-modules is a $\mathcal{C}$ natural transformation [Bor94, 6.2.4]. A map of $\mathcal{O}$-modules $f: X \longrightarrow Y$ is 
an objectwise equivalence (or objectwise fibration) if $f(i): X(i) \longrightarrow Y(i)$ is a weak equivalence (fibration) in $\mathcal{C}$ for each all $i \in I$. A cofibration is a map with the left lifting property with respect to any trivial fibration. For every element $j \in I$, there is a free or representable $\mathcal{O}$-module $F_{j}^{\mathcal{O}}$ defined by $F_{j}^{\mathcal{O}}(i)=\mathcal{O}(i, j)$. As the name suggests, homomorphisms from $F_{j}^{\mathcal{O}}$ into a module $M$ are in bijective correspondence with morphisms from $\mathbb{I}$ to $M(j)$ by the enriched Yoneda Lemma [Bor94, 6.3.5]. The evaluation functor at $j$, $\mathrm{Ev}_{j}: \mathcal{M o d}-\mathcal{O} \longrightarrow \mathcal{C}$ has a left adjoint 'free' functor which sends an object $X$ of $\mathcal{C}$ to the module $F_{j}^{\mathcal{O}} X=X \otimes F_{j}^{\mathcal{O}}$, where $X \otimes-$ is defined by tensoring objectwise with $X$.

A morphism $\Psi: \mathcal{O} \longrightarrow \mathcal{R}$ of $\mathcal{C} I$-categories is simply a $\mathcal{C}$-functor which is the identity on objects. We denote by $\mathcal{C} I-\mathcal{C}$ at the category of all $\mathcal{C} I$-categories. The restriction of scalars

$$
\Psi^{*}: \operatorname{Mod}-\mathcal{R} \longrightarrow \mathcal{M o d}-\mathcal{O} \quad ; \quad M \longmapsto M \circ \Psi
$$

has a left adjoint functor $\Psi_{*}$, also denoted $-\otimes_{\mathcal{O}} \mathcal{R}$, which we refer to as extension of scalars. As usual it is given by an enriched coend, i.e., for an $\mathcal{O}$-module $M$ the $\mathcal{R}$-module $\Psi_{*} M=M \otimes_{\mathcal{O}} \mathcal{R}$ is given by the coequalizer of the two $\mathcal{R}$-module homomorphisms

$$
\bigvee_{i, j \in I} M(j) \otimes \mathcal{O}(i, j) \otimes F_{i}^{\mathcal{R}} \Longrightarrow \bigvee_{i \in I} M(i) \otimes F_{i}^{\mathcal{R}}
$$

We call $\Psi: \mathcal{O} \longrightarrow \mathcal{R}$ a (pointwise) weak equivalence of $\mathcal{C} I$-categories if the $\mathcal{C}$ morphism $\Psi_{i, j}: \mathcal{O}(i, j) \longrightarrow \mathcal{R}(i, j)$ is a weak equivalence for all $i, j \in I$, . Next we establish the model category structure for $\mathcal{O}$-modules and discuss Quillen invariance for weak equivalences of $\mathcal{C}$-categories.

Theorem 6.1 Let $\mathcal{C}$ be a cofibrantly generated monoidal model category which satisfies the monoid axiom [SSO0, 3.3] and such that every object of $\mathcal{C}$ is small relative to the whole category.

(1) Let $\mathcal{O}$ be a $\mathcal{C} I$-category. Then the category of $\mathcal{O}$-modules with the objectwise equivalences, objectwise fibrations, and cofibrations is a cofibrantly generated model category.

(2) Let $\Psi: \mathcal{O} \longrightarrow \mathcal{R}$ be a weak equivalence of $\mathcal{C} I$-categories. Suppose that for every cofibrant right $\mathcal{O}$-module $N$, the induced map $N \otimes_{\mathcal{O}} \mathcal{O} \longrightarrow$ $N \otimes_{\mathcal{O}} \mathcal{R}$ is an objectwise weak equivalence. Then restriction and extension of scalars along $\Psi$ form a $\mathcal{C}$-Quillen equivalence of the module categories.

Proof We use [SS00, 2.3] to establish the model category for $\mathcal{O}$-modules. Let $\mathbb{I}_{I}$ denote the initial $\mathcal{C} I$-category with $\mathbb{I}_{I}(i, i)=\mathbb{I}_{\mathcal{C}}$ and $\mathbb{I}_{I}(i, j)=\emptyset$, the 
initial object, for $i \neq j$. The category of $\mathbb{I}_{I}$-modules is the $I$-indexed product category of copies of $\mathcal{C}$. Hence it has a cofibrantly generated model category inherited from $\mathcal{C}$ in which the cofibrations, fibrations and weak equivalences are objectwise. Here the generating trivial cofibrations are the generating trivial cofibrations of $\mathcal{C}$ between objects concentrated at one object, i.e. of the form $A_{j}$ with $A_{j}(j)=A$ and $A_{j}(i)=\emptyset$ if $i \neq j$.

The unit morphism $\mathbb{I}_{I} \longrightarrow \mathcal{O}$ induces adjoint functors of restriction and extension of scalars between the module categories. This produces a triple $-\otimes_{\mathbb{I}_{I}} \mathcal{O}$ on $\mathbb{I}_{I}$-modules with the algebras over this triple the $\mathcal{O}$-modules. Then the generating trivial cofibrations for $\mathcal{O}$-modules are maps between modules of the form $A_{j} \otimes_{\mathbb{I}_{I}} \mathcal{O}=A \otimes \mathcal{O}(-, j)$. Hence the monoid axiom applies to show that the new generating trivial cofibrations and their relative cell morphisms are weak equivalences. Thus, since all objects in $\mathcal{C}$ are assumed to be small, the model category structure follows by criterion (1) of [SS00, 2.3].

The proof of Part (2) follows as in [SSO0, 4.3]. The restriction functor $\Psi^{*}$ preserves objectwise fibrations and objectwise equivalences, so restriction and extension of scalars form a Quillen adjoint pair. By assumption, for $N$ a cofibrant right $\mathcal{O}$-module

$$
N \cong N \otimes_{\mathcal{O}} \mathcal{O} \longrightarrow N \otimes_{\mathcal{O}} \mathcal{R}
$$

is a weak equivalence. Thus if $M$ is a fibrant right $\mathcal{R}$-module, an $\mathcal{O}$-module map $N \longrightarrow \Psi^{*} M$ is a weak equivalence if and only if the adjoint $\mathcal{R}$-module map $\Psi_{*} N=N \otimes_{\mathcal{O}} \mathcal{R} \longrightarrow M$ is a weak equivalence.

\subsection{Categories as monoids of graphs}

In [ML71, II.7] Mac Lane explains how a small category with object set $I$ can be viewed as a monoid in the category of $I$-indexed graphs. We need an enriched version of this giving $\mathcal{C}$-categories as the monoids with respect to a monoidal product on the category of $\mathcal{C}$-graphs, so that we can apply the general theory of [SS00]. Note that here the product on $\mathcal{C}$-graphs is not symmetric monoidal, so we must take care in applying [SS00].

Let $\left(\mathcal{C}, \otimes, \mathbb{I}_{\mathcal{C}}\right)$ be a closed symmetric monoidal closed category with an initial object $\emptyset$. Let $I$ be any set. The category of (directed) $I$-graphs in $\mathcal{C}$, denoted $\mathcal{C} I-\mathcal{G}$ raph is simply the product category of copies of $\mathcal{C}$ indexed by the set $I \times I$. If $G$ is an $I$-graph in $\mathcal{C}$, then one can think of $G(i, j)$ as the $\mathcal{C}$-object of arrows pointing from the vertex $i$ to the vertex $j$. 
If $G$ and $H$ are two $I$-graphs, then their tensor product is defined by the formula

$$
(G \otimes H)(i, j)=\bigvee_{k \in I} G(k, j) \otimes H(i, k) .
$$

The $I$-graph $\mathbb{I}_{I}$ is defined by

$$
\mathbb{I}_{I}(i, j)= \begin{cases}\mathbb{I} & \text { if } i=j \\ \emptyset & \text { if } i \neq j\end{cases}
$$

The smash product makes the category of $I$-graphs in $\mathcal{C}$ into a monoidal category with unit object $\mathbb{I}_{I}$ (but not a symmetric monoidal category). Moreover, the category $\mathcal{C} I$ - $\mathcal{C}$ at of $\mathcal{C} I$-categories is precisely the category of monoids in $\mathcal{C} I-\mathcal{G}$ raph with respect to the smash product. Note that when $I$ is a singleton set $\mathcal{C} I$ - $\mathcal{G}$ raph is $\mathcal{C}$ and $\mathcal{C} I$-C at is $\mathcal{C}$-Monoid.

Warning There is a slight risk of confusion in the notion of a module over a $\mathcal{C} I$-category when $I$ has more than one element. As we just explained, such a $\mathcal{C} I$-category $\mathcal{O}$ is a monoid with respect to the monoidal product (6.2) of $I$ graphs. So there is a notion of $\mathcal{O}$-module which is an $I$-graph $M$ together with a morphism of $I$-graphs $M \otimes \mathcal{O} \longrightarrow M$ which satisfies associativity and unit constraints. However, this is not the same as the $\mathcal{O}$-modules defined above as the enriched functors from $\mathcal{O}$ to $\mathcal{C}$. These enriched functors have an underlying $I$-indexed family of objects in $\mathcal{C}$, whereas the other kind of modules have an underlying $I$-graph, so they have underlying $\mathcal{C}$-objects indexed by ordered pairs of elements from $I$. However, if $M$ is an $I$-graph with an associative, unital $I$-graph morphism $M \otimes \mathcal{O} \longrightarrow M$, then we can fix an element $j \in I$, and obtain an enriched functor $M(-, j)$. So the modules which have underlying $I$-graphs give rise to an $I$-indexed family of modules in the earlier sense.

Since $\mathcal{C} I-\mathcal{G}$ raph is not a symmetric monoidal category, the results of [SS00] do not apply directly to produce a model category on the monoids, $\mathcal{C} I-\mathcal{C}$ at. It turns out though that the proof Theorem 4.1 of [SS00] carries over since the homotopy type of a graph is determined pointwise and $\mathcal{C}$ is assumed to be symmetric monoidal. First, if $\mathcal{C}$ is a cofibrantly generated model category, then $\mathcal{C} I-\mathcal{G}$ raph is also a cofibrantly generated model category with the cofibrations, fibrations and weak equivalences defined pointwise, i.e., for each $(i, j)$. The generating (trivial) cofibrations are of the form $A_{i, j} \longrightarrow B_{i, j}$ where $A \longrightarrow B$ is a generating (trivial) cofibration in $\mathcal{C}$ and $A_{i, j}$ is the $\mathcal{C} I-\mathcal{G}$ raph with value $A$ concentrated at $(i, j)$. Based on this model category we use [SS00, $4.1(3)]$ to construct a model category on the associated category of monoids, $\mathcal{C} I$ - $\mathcal{C}$ at . 
Dundas [Du01, Thm. 3.3] obtains the model structure on the category $\mathcal{C} I$-C at of $\mathcal{C} I$-categories under slightly different assumptions, namely when the underlying monoidal model category $\mathcal{C}$ is simplicial and has a monoidal fibrant replacement functor. Part (2) of the following Proposition is essentially the same as Lemma 3.6 of [Du01].

Proposition 6.3 Let $I$ be a fixed set and $\mathcal{C}$ be a cofibrantly generated monoidal model category which satisfies the monoid axiom. Assume as well that every object in $\mathcal{C}$ is small.

(1) $\mathcal{C} I-\mathcal{C}$ at is a cofibrantly generated model category with weak equivalences and fibrations defined pointwise.

(2) Every cofibration of $\mathcal{C} I$-categories whose source is pointwise cofibrant is a pointwise cofibration. In particular, if the unit object $\mathbb{I}_{\mathcal{C}}$ is cofibrant in $\mathcal{C}$, then every cofibrant $\mathcal{C} I$ category is pointwise cofibrant.

Proof The generating (trivial) cofibrations are the image of the free monoid functor $T_{\mathcal{C} I}: \mathcal{C} I-\mathcal{G}$ raph $\longrightarrow \mathcal{C} I-\mathcal{C}$ at applied to the sets of generating (trivial) cofibrations in $\mathcal{C} I-\mathcal{G}$ raph. The proof of the first statement follows from [SS00, $4.1(3), 6.2]$. The third to last paragraph in the proof of [SS00, 6.2] is the only place which uses the symmetry of the monoidal product. At that point one is working in the underlying category, which is $\mathcal{C} I-\mathcal{G}$ raph in our case. Since both weak equivalences and pushouts in $\mathcal{C} I-\mathcal{G}$ raph are determined pointwise, one can just work pointwise in $\mathcal{C}$. Then the symmetry of the monoidal product and the monoid axiom do hold by assumption.

The proof of the second statement is essentially the same as in the last paragraph of [SS00, 6.2], which treats the case of algebras, i.e., when $I$ is a singleton. Note, this analysis does not require a symmetric monoidal product.

As with Theorem 3.12 we use the following comparison of $\lambda$ with the structured left adjoints $L^{\mathcal{C} I}$ and $L_{\mathcal{O}}$ for categories, respectively modules. In this paper we only apply Theorem 6.5 to strong monoidal Quillen pairs, namely in the next and final section. In that case, the maps $\chi_{\mathcal{O}}$ and $\chi_{M}$ are isomorphisms, and so the proposition is redundant. Elsewhere this statement is used for weak monoidal Quillen pairs, though; see [S]. Refer to Definition 3.2 for the notion of a 'cell' object.

Proposition 6.4 Let $(\lambda: \mathcal{D} \longleftrightarrow \mathcal{C}: R)$ be a weak monoidal Quillen pair between monoidal model categories with cofibrant unit objects. 
(1) Suppose that the functor $R: \mathcal{C} I-\mathcal{C}$ at $\longrightarrow \mathcal{D} I-\mathcal{C}$ at has a left adjoint $L^{\mathcal{C} I}$. Then for every $\mathcal{D} I$-cell category $\mathcal{O}$, the morphism of $\mathcal{C} I$-graphs

$$
\chi_{\mathcal{O}}: \lambda \mathcal{O} \longrightarrow L^{\mathcal{C} I} \mathcal{O}
$$

which is adjoint to the underlying $\mathcal{D} I$-graph morphism of the adjunction unit $\mathcal{O} \longrightarrow R\left(L^{\mathcal{C I}} \mathcal{O}\right)$ is a pointwise weak equivalence.

(2) Suppose $\mathcal{O}$ is a $\mathcal{D} I$-cell category for which the functor $R: \mathcal{M o d}-L^{\mathcal{C} I} \mathcal{O} \longrightarrow$ $\mathcal{M}$ od- $\mathcal{O}$ has a left adjoint $L_{\mathcal{O}}$. Then for every cell $\mathcal{O}$-module $M$, the map

$$
\chi_{M}: \lambda M \longrightarrow L_{\mathcal{O}} M
$$

which is adjoint to the underlying $\mathcal{D}$-morphism of the adjunction unit $M \longrightarrow R\left(L_{\mathcal{O}} M\right)$ is an objectwise weak equivalence in $\mathcal{C}$.

We omit the proof of Proposition 6.4, since it is essentially identical to the proof of Proposition 5.1; the free monoid functor is replaced by the free $I$-category functor $T_{\mathcal{C} I}: \mathcal{C} I-\mathcal{G}$ raph $\longrightarrow \mathcal{C} I-\mathcal{C}$ at and the underlying objects are in $\mathcal{C} I-\mathcal{G}$ raph and $\mathcal{D} I$-Graph instead of $\mathcal{C}$ and $\mathcal{D}$. For modules there is another difference: instead of one kind of free module, for every element $j \in I$ and every object $K$ of $\mathcal{C}$, there is an $\mathcal{O}$-module $F_{j} K=K \otimes \mathcal{O}(-, j)$ freely generated by $K$ at $j$.

Finally, we extend the results of Section 3 to these enriched categories.

Theorem 6.5 Let $R: \mathcal{C} \longrightarrow \mathcal{D}$ be the right adjoint of a weak monoidal Quillen equivalence. Suppose that the unit objects in $\mathcal{C}$ and $\mathcal{D}$ are cofibrant.

(1) Consider a cofibrant $\mathcal{D} I$ category $\mathcal{O}$ such that the forgetful functors create model structures for modules over $\mathcal{O}$ and modules over $L^{\mathcal{C} I} \mathcal{O}$. Then the adjoint functor pair

$$
L_{\mathcal{O}}: \operatorname{Mod}-\mathcal{O} \rightleftarrows \operatorname{Mod}-\left(L^{\mathcal{C I}} \mathcal{O}\right): R
$$

is a Quillen equivalence.

(2) Suppose that Quillen invariance holds for $I$-categories in $\mathcal{C}$ and $\mathcal{D}$. Then for any pointwise fibrant $\mathcal{C} I$-category $\mathcal{A}$ such that the forgetful functors create model structures for modules over $\mathcal{A}$ and modules over $R \mathcal{A}$, the adjoint functor pair

$$
L^{\mathcal{A}}: \operatorname{Mod}-R \mathcal{A} \longleftrightarrow \operatorname{Mod}-\mathcal{A}: R
$$

is a Quillen equivalence. If the right adjoint $R$ preserves all weak equivalences in $\mathcal{C}$ and the forgetful functors create model structures for modules over any monoid, then this holds for any $\mathcal{C} I$-category $\mathcal{A}$.

The proof of Theorem 6.5 is now almost literally the same as the proof of parts (1) and (2) of Theorem 3.12. Whenever Proposition 5.1 is used in the proof of the latter we now appeal to Proposition 6.4 instead. 


\section{Symmetric monoidal categories of spectra}

In this section we show that the Quillen equivalences between the categories of rings, modules and algebras established in [MMSS] and [Sch01] can be extended to Quillen equivalences between modules over 'ring spectra with many objects' or 'spectral categories'. This then shows that the classification results in [SS03] can be translated to any one of the symmetric monoidal categories of spectra.

Comparison theorems between rings, modules and algebras based on symmetric spectra over simplicial sets $\left(S p_{s S}^{\Sigma}\right)$ and topological spaces $\left(S p_{T o p}^{\Sigma}\right)$, orthogonal spectra $(\mathcal{I S}), \mathcal{W}$-spaces $(\mathcal{W} \mathcal{T})$ and simplicial functors $(\mathcal{S F})$ can be found in [MMSS], Theorems 0.4 through 0.9 and 19.11. Rings, modules and algebras based on $S$-modules $\mathcal{M}_{S}$ are compared to their counterparts based on symmetric and orthogonal spectra in [Sch01, Thm. 5.1] respectively [MM02, Ch. I], Theorems 1.1 through 1.7. See [Sh01] for an approach that unifies all of these comparison theorems. These Quillen equivalences could also be deduced via our general result in Theorem 3.12. Moreover, in several of the categories which participate in the diagram (7.1) below, there are also Quillen equivalences for categories of commutative algebras (with the exception of simplicial functors $\mathcal{S F}$ and $\mathcal{W}$-spaces $\mathcal{W T}$ ); such results are out of the scope of the general methods in this present paper. However, modules over 'ring spectra with many objects' were not considered in the above papers, and the point of this final section is to fill that gap.

The categories of spectra which we consider are all displayed in a commutative diagram of monoidal model categories and strong monoidal Quillen equivalences

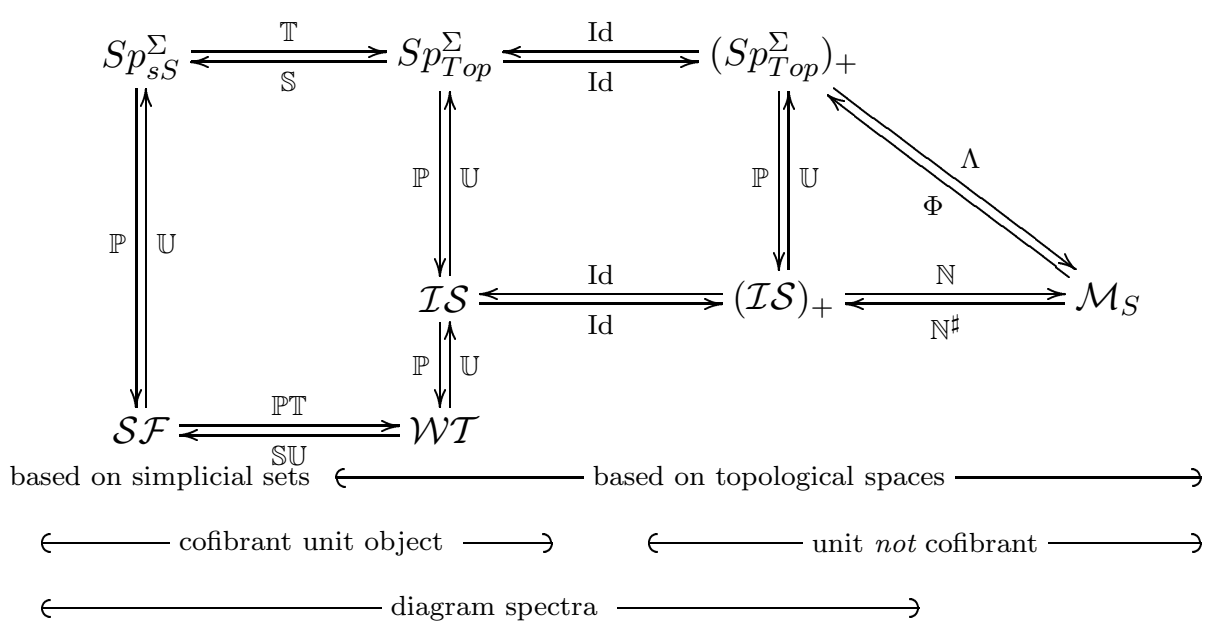

Algebraic 83 Geometric Topology, Volume 3 (2003) 
(where the left adjoints are on top and on the left). There are five categories of diagram spectra: symmetric spectra over simplicial sets $S p_{s S}^{\Sigma}$ [HSS], symmetric spectra over topological spaces $S p_{\text {Top }}^{\Sigma}$ [MMSS], simplicial functors $\mathcal{S F}$ [Lyd98], orthogonal spectra $\mathcal{I S}$ [MMSS], and $\mathcal{W}$-spaces $\mathcal{W} \mathcal{T}$ [MMSS]. The categories of topological symmetric spectra and orthogonal spectra appear twice, with different model structures: the stable model structure [HSS, 3.4], [MMSS, Sec. 9] (without decoration) and the positive stable model structure [MMSS, Sec. 14] (decorated with a subscript ' + '). However, the stable and positive model structures share the same class of weak equivalences. The remaining category $\mathcal{M}_{S}$ of $S$-modules [EKMM] is of a somewhat different flavor. By $\mathbb{U}$ we denote various 'forgetful' or 'underlying object' functors, with the left adjoint 'prolongation' functors $\mathbb{P}$, which are all described in the paper [MMSS]. Moreover, $\mathbb{S}$ is the singular complex functor and $\mathbb{T}$ is geometric realization. The functors $\Lambda$ and $\Phi$ which relate symmetric spectra to $S$-modules and their lifts $\mathbb{N}$ and $\mathbb{N} \sharp$ to orthogonal spectra are defined and studied in [Sch01] respectively [MM02, Ch. I]. See [Sh01, 4.7] for a unified approach to defining all of these functors.

The following theorem is an application of Theorem 6.1 to these categories of spectra.

Theorem 7.2 Let $\mathcal{C}$ be any of the model categories $S p_{s S}^{\Sigma}, S p_{\text {Top }}^{\Sigma},\left(S p_{\text {Top }}^{\Sigma}\right)_{+}$, $\mathcal{I S},(\mathcal{I S})_{+}, \mathcal{S F}, \mathcal{W T}$ or $\mathcal{M}_{S}$

(1) The modules over any $\mathcal{C}$-category inherit a model category structure in which the fibrations and weak equivalences are defined pointwise in the underlying category $\mathcal{C}$.

(2) If $\Psi: \mathcal{O} \longrightarrow \mathcal{R}$ is a pointwise weak equivalence of $\mathcal{C} I$-categories, then restriction and extension of scalars along $\Psi$ form a $\mathcal{C}$-Quillen equivalence of the module categories.

Proof (1) All specified choices of monoidal model category $\mathcal{C}$ are cofibrantly generated, see [MMSS, 12.1], [HSS, 3.4], [Lyd98, 9.2] and [EKMM, VII.4]. For $\mathcal{M}_{S}$ the argument for (1) follows just as for modules over a ring spectrum; see [EKMM, VII.4.7]. One could also verify the pushout product, unit and monoid axioms directly. The unit axiom is automatic for the categories $S p_{s S}^{\Sigma}$, $S p_{\text {Top }}^{\Sigma}, \mathcal{I S}, \mathcal{S F}$ and $\mathcal{W} \mathcal{T}$ where the unit object is cofibrant. In the positive model structures $\left(S p_{\text {Top }}^{\Sigma}\right)_{+}$and $(\mathcal{I S})_{+}$the unit axiom holds since every positively cofibrant object is also stably cofibrant, and the respective unit objects are stably (but not positively) cofibrant. Moreover, the pushout product and monoid axioms for the diagram spectra other than $\mathcal{S F}$ hold by [MMSS, 12.5, 
12.6], [HSS, 5.3.8, 5.4.1]. For the category of simplicial functors, these two axioms do not appear explicitly in Lydakis' paper [Lyd98], but we can argue as follows.

By [Lyd98, 12.3] the pushout product of two cofibrations is a cofibration. To see that the pushout product $i \square j$ of a cofibration $i$ with a trivial cofibration $j$ is again a trivial cofibration, we argue indirectly and use the Quillen equivalence $\mathbb{P T}: \mathcal{S F} \longrightarrow \mathcal{W} \mathcal{T}$ of [MMSS, 19.11]. By [SS00, 3.5 (1)] or [Hov99, 4.2.5] it suffices to check the pushout product of a generating cofibration with a generating trivial cofibration. For the stable model structure of simplicial functors, Lydakis [Lyd98, 9.1] uses generating cofibrations and trivial cofibrations which all have cofibrant sources and targets. Since the left Quillen functor $\mathbb{P} \mathbb{T}$ is also strong monoidal, we have $\mathbb{P} \mathbb{T}(i \square j) \cong(\mathbb{P} T i) \square(\mathbb{P T} j)$ as morphisms of $\mathcal{W}$-spaces. Since $\mathbb{P} \mathbb{T} i$ is a cofibration, $\mathbb{P} \mathbb{T} j$ is a trivial cofibration and the pushout product axiom holds in $\mathcal{W} \mathcal{T}$, the pushout product $\mathbb{P T}(i \square j)$ is in particular a stable equivalence of $\mathcal{W}$-spaces. As a left Quillen equivalence, $\mathbb{P T}$ detects weak equivalences between cofibrant objects, so $i \square j$ is a stable equivalence of simplicial functors.

For the monoid axiom we consider a generating stable trivial cofibration $j$ from the set $\mathbf{S F}_{\text {sac }}^{g}$ defined in [Lyd98, 9.1], and we let $X$ be an arbitrary simplicial functor. By [Lyd98, 12.3], $X \wedge j$ is an injective morphism of simplicial functors; we claim that $X \wedge j$ is also a stable equivalence. To see this, we choose a cofibrant replacement $X^{c} \longrightarrow X$; then $X^{c} \wedge j$ is a weak equivalence by the pushout product axiom. By [Lyd98, 12.6], smashing with a cofibrant simplicial functor preserves stable equivalences. Since the source and target of $j$ are cofibrant, $X \wedge j$ is thus also a stable equivalence. The class of injective stable equivalences of simplicial functors is closed under cobase change and transfinite

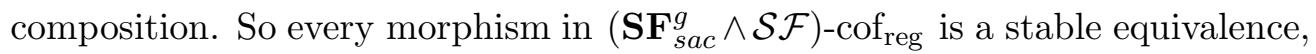
which implies the monoid axiom by [SSO0, $3.5(2)]$.

In the categories $S p_{s S}^{\Sigma}$ and $\mathcal{S F}$ which are based on simplicial sets, every object is small with respect to the whole category; so the proof concludes by an application of Theorem 6.1 (1). In the other cases, which are based on topological spaces, the cofibration hypothesis [MMSS, 5.3], [EKMM, VII.4] makes sure that the small object argument still applies and the conclusion of Theorem 6.1 is still valid.

Part (2) is proved by verifying the criterion of Theorem 6.1 (2): for every cofibrant right $\mathcal{O}$-module $N$, the induced map $N \otimes_{\mathcal{O}} \mathcal{O} \longrightarrow N \otimes_{\mathcal{O}} \mathcal{R}$ is an objectwise weak equivalence. The special case of modules over a monoid, i.e., when the set $I$ has one element, is treated in [MMSS, 12.7], [HSS, 5.4.4] and [EKMM, 
III 3.8]. Again for simplicial functors, this argument is not quite contained in [Lyd98], but one can also verify the criterion for Quillen invariance as in [MMSS, 12.7] using the fact that smashing with a cofibrant object preserves stable weak equivalences by $[$ Lyd98, 12.6]. The general case of modules over a category with more than one object uses the same kind of cell induction as for modules over a monoid; we omit the details.

All the Quillen adjoint pairs appearing in the master diagram (7.1) are strong monoidal Quillen equivalences in the sense of Definition 3.6; so we can apply Theorem 6.5 to get Quillen equivalences for modules over 'ring spectra with many objects'; this leads to the following proof of Corollary 1.2. Special care has to be taken for the positive model structures on the categories of symmetric and orthogonal spectra, and for the category of $S$-modules since there the units of the smash product are not cofibrant.

Proof of Corollary 1.2 One of the main results of [SS03], Theorem 3.3.3, shows that any cofibrantly generated, proper, simplicial stable model category with a set of generators is Quillen equivalent to modules over a $S p_{s S^{\Sigma}}^{\Sigma}$-category $\mathcal{O}$ with one object for each generator. By Proposition 6.3 we can choose a cofibrant replacement $S p_{s S^{\Sigma}}^{\Sigma}$-cell category $\mathcal{O}^{c}$ with a pointwise stable equivalence $q: \mathcal{O}^{c} \stackrel{\sim}{\longrightarrow} \mathcal{O}$. Then $\mathcal{M o d}-\mathcal{O}$ and $\mathcal{M o d}-\mathcal{O}^{c}$ are Quillen equivalent.

For comparisons which do not involve the category $\mathcal{M}_{S}$ of $S$-modules, but only the left part of diagram (7.1), we consider the stable model structures. In the five categories of diagram spectra, the unit of the smash product is cofibrant with respect to this stable model structure. So various applications of part (1) of Theorem 6.5 show that $\operatorname{Mod}-\mathcal{O}^{c}$ is Quillen equivalent to modules over the $S p_{\text {Top }}^{\Sigma}$-category $\mathbb{T}\left(\mathcal{O}^{c}\right)$ (where $\mathbb{T}$ is the geometric realization functor, applied levelwise), to modules over the $\mathcal{I} \mathcal{S}$-category $\mathbb{P} \mathbb{T}\left(\mathcal{O}^{c}\right)$, to modules over the $\mathcal{W} \mathcal{T}$ category $\mathbb{P P T}\left(\mathcal{O}^{c}\right)$ (this composite $\mathbb{P P}$ is just denoted by $\mathbb{P}$ in [MMSS]), and to modules over the $\mathcal{S} \mathcal{F}$-category $\mathbb{P}\left(\mathcal{O}^{c}\right)$.

To connect to the world $\mathcal{M}_{S}$ of $S$-modules we have to argue slightly differently, since the unit $S$ in $\mathcal{M}_{S}$ is not cofibrant. First we change model structures on the category of $\mathbb{T}\left(\mathcal{O}^{c}\right)$-modules by viewing the identity functors as a Quillen equivalence between the stable and positive model structures (which share the same class of weak equivalences); see the right-hand part of the diagram below.

The last Quillen pair we consider compares modules over $\mathbb{T}\left(\mathcal{O}^{c}\right)$ and modules over the $\mathcal{M}_{S}$-category $\Lambda \mathbb{T}\left(\mathcal{O}^{c}\right)$. The right adjoint is given by $\Phi$, together with restriction of scalars along the adjunction unit $\eta: \mathbb{T}\left(\mathcal{O}^{c}\right) \longrightarrow \Phi \Lambda \mathbb{T}\left(\mathcal{O}^{c}\right)$; the left 
adjoint is induced by pointwise application of $\Lambda$. The right adjoint $\Phi$ preserves all weak equivalences; so to see that we have a Quillen equivalence we may show that for every cofibrant $\mathbb{T}\left(\mathcal{O}^{c}\right)$-module $M$ the adjunction unit $M \longrightarrow \Phi \Lambda(M)$ is a pointwise stable equivalence. Since $\mathcal{O}^{c}$ is a $S p_{s S^{\Sigma}}^{\Sigma}$-cell category, $\mathbb{T}\left(\mathcal{O}^{c}\right)$ is a $S p_{\text {Top }}^{\Sigma}$-cell category, both times with respect to the stable model structure on symmetric spectra. The unit is cofibrant in the stable model structure of symmetric spectra; hence $\mathbb{T}\left(\mathcal{O}^{c}\right)$ is pointwise cofibrant in the stable model structure of symmetric spectra as well.

The positive cofibrations of symmetric spectra are precisely those stable cofibration which are isomorphisms in level 0 . So every positively cofibrant $\mathbb{T}\left(\mathcal{O}^{c}\right)$ module $M$ is also stably cofibrant. Since $\mathbb{T}\left(\mathcal{O}^{c}\right)$ itself is is pointwise stably cofibrant, so is $M$; thus the adjunction unit $M \longrightarrow \Phi \Lambda(M)$ is a stable equivalence by [Sch01, Thm. 3.1].

To sum up, we display all these Quillen equivalences in the following diagram, where we also indicate the underlying monoidal model categories:

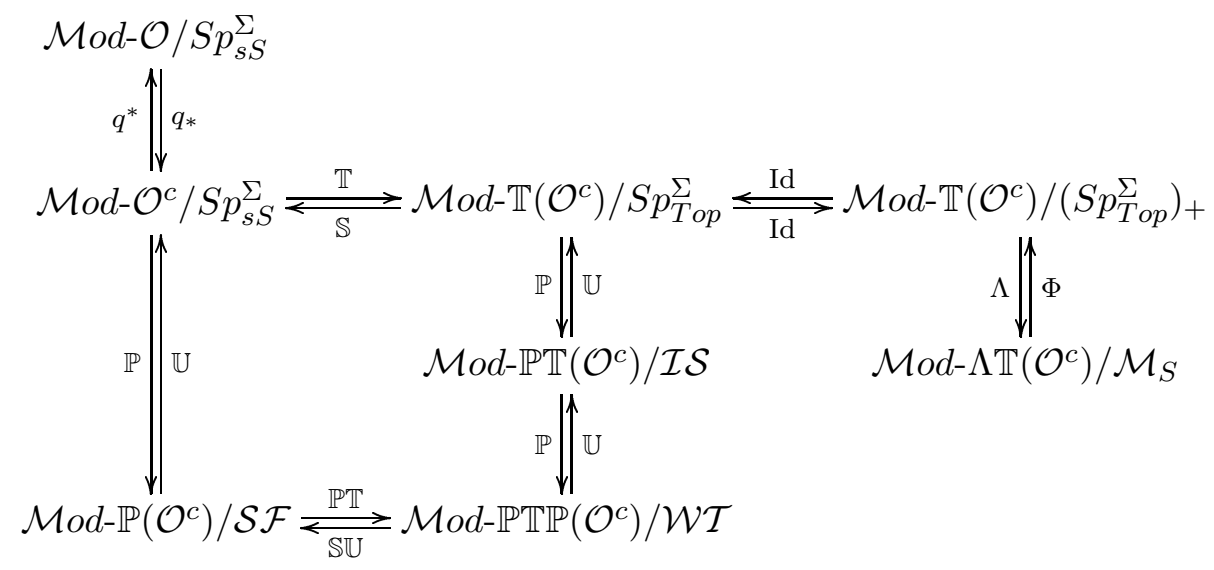

\section{References}

[BM] C. Berger and I. Moerdijk, Axiomatic homotopy theory for operads, Preprint (2002). http://arXiv.org/abs/math. AT/0206094

[Bor94] F. Borceux, Handbook of categorical algebra II, Categories and structures, Cambridge University Press, 1994.

[BG76] A. K. Bousfield and V. K. A. M. Gugenheim, On PL de Rham theory and rational homotopy type, Mem. Amer. Math. Soc. 8 (1976), no. 179, ix+94 pp.

[Ca54] H. Cartan, Algèbres d'Eilenberg-MacLane at homotopie, Séminaire Henri Cartan, $1954-55$

Algebraic 83 Geometric Topology, Volume 3 (2003) 
[Do58] A. Dold, Homology of symmetric products and other functors of complexes, Ann. Math. 68 (1958), 54-80.

[Du01] B. I. Dundas, Localization of V-categories, Theory Appl. Categ. 8 (2001), 284-312.

[Dw80] W. G. Dwyer, Homotopy operations for simplicial commutative algebras. Trans. Amer. Math. Soc. 260 (1980), 421-435.

[DK80] W. G. Dwyer and D. M. Kan, Function complexes in homotopical algebra, Topology 19 (1980), 427-440.

[DS95] W. G. Dwyer and J. Spalinski, Homotopy theories and model categories, Handbook of algebraic topology (Amsterdam), North-Holland, Amsterdam, 1995, pp. $73-126$.

[EM53] S. Eilenberg and S. Mac Lane, On the groups $H(\Pi, n), I$, Ann. of Math. (2) 58, (1953), 55-106.

[EKMM] A. D. Elmendorf, I. Kriz, M. A. Mandell, and J. P. May, Rings, modules, and algebras in stable homotopy theory. With an appendix by M. Cole, Mathematical Surveys and Monographs, 47, Amer. Math. Soc., Providence, RI, 1997, xii+249 pp.

[GS] J. P. C. Greenlees and B. Shipley, Rational torus-equivariant cohomology theories III: the Quillen equivalence, in preparation.

[Hov99] M. Hovey, Model categories, Mathematical Surveys and Monographs, 63, Amer. Math. Soc., Providence, RI, 1999, xii+209 pp.

[HSS] M. Hovey, B. Shipley, and J. Smith, Symmetric spectra, J. Amer. Math. Soc. 13 (2000), 149-208.

[Jar97] J. F. Jardine, A closed model structure for differential graded algebras, Cyclic Cohomology and Noncommutative Geometry, Fields Institute Communications, 17, AMS (1997), 55-58.

[Lyd98] M. Lydakis, Simplicial functors and stable homotopy theory, Preprint (1998). http://hopf.math.purdue.edu/

[ML63] S. Mac Lane, Homology, Grundlehren der math. Wissensch. 114, Academic Press, Inc., Springer-Verlag, $1963 \mathrm{x}+422 \mathrm{pp}$.

[ML71] S. Mac Lane, Categories for the working mathematician, Graduate Texts in Math. 5, Springer, New York-Berlin, 1971, ix +262 pp.

[Man] M. A. Mandell, Topological Andre-Quillen Cohomology and E-infinity AndreQuillen Cohomology, Adv. in Math., to appear.

http://www.math.uchicago.edu/ ${ }^{\text {mandell/ }}$

[MMSS] M. A. Mandell, J. P. May, S. Schwede and B. Shipley, Model categories of diagram spectra, Proc. London Math. Soc., 82 (2001), 441-512.

[MM02] M. A. Mandell and J. P. May, Equivariant orthogonal spectra and S-modules, Memoirs Amer. Math. Soc., 159 (2002), no. 755, x+108 pp. 
[May67] J. P. May, Simplicial objects in algebraic topology, Chicago Lectures in Mathematics, Chicago, 1967, viii+161pp.

[Qui67] D. G. Quillen, Homotopical algebra, Lecture Notes in Mathematics, 43, Springer-Verlag, 1967.

[Qui69] D. G. Quillen, Rational homotopy theory, Ann. of Math. 90 (1969), 204-265.

[Ri03] B. Richter, Symmetries of the Dold-Kan correspondence, Math. Proc. Cambridge Phil. Soc. 134 (2003), 95-102.

[Sch01] S. Schwede, S-modules and symmetric spectra, Math. Ann. 319 (2001), 517532.

[SS00] S. Schwede and B. Shipley, Algebras and modules in monoidal model categories, Proc. London Math. Soc. 80 (2000), 491-511.

[SS03] S. Schwede and B. Shipley, Stable model categories are categories of modules, Topology, 42 (2003), 103-153.

[Sh01] B. Shipley, Monoidal uniqueness of stable homotopy theory, Adv. in Math. 160 (2001), 217-240.

[Sh02] B. Shipley, An algebraic model for rational $S^{1}$-equivariant stable homotopy theory, Quart. J. of Math. 53 (2002), 87-110.

[S] B. Shipley, HZZ-algebra spectra are differential graded algebras, Preprint (2002). http://www . math.purdue.edu/ bshipley/

[St] D. Stanley, Determining closed model category structures, Preprint (1998). http://hopf . math.purdue.edu/

SFB 478 Geometrische Strukturen in der Mathematik

Westfälische Wilhelms-Universität Münster, Germany

Department of Mathematics, Purdue University

W. Lafayette, IN 47907, USA

Email: sschwede@math.uni-muenster.de and bshipley@math.purdue.edu

Received: 18 August 2002 Revised: 11 February 2003

Algebraic 63 Geometric Topology, Volume 3 (2003) 